\title{
On peaceful coexistence: is the collapse postulate incompatible with relativity?
}

\author{
Wayne C. Myrvold
}

Department of Philosophy, University of Western Ontario, London, Ont., Canada N6A 3 K7

\begin{abstract}
In this paper, it is argued that the prima facie conflict between special relativity and the quantum-mechanical collapse postulate is only apparent, and that the seemingly incompatible accounts of entangled systems undergoing collapse yielded by different reference frames can be regarded as no more than differing accounts of the same processes and events. Attention to the transformation properties of quantum-mechanical states undergoing unitary, non-collapse evolution points the way to a treatment of collapse evolution consistent with the demands of relativity. (C) 2002 Elsevier Science Ltd. All rights reserved.
\end{abstract}

Keywords: Relativity; Quantum theory; Peaceful coexistence; State vector reduction; Maudlin; Fleming

\section{Introduction}

There is a strong prima facie case for a claim that the reduction of the quantummechanical state vector upon measurement, construed as a physical process, is incompatible with the special theory of relativity. Suppose, for example, that a position measurement is performed on a particle whose wave function extends throughout a large region of space. The collapse postulate holds that, after the measurement, the particle is located in the region in which it is found, and hence that the value of the wave function everywhere else has instantaneously gone to zero. If such a process is regarded as a physical event, and not as a mere shift in perspective or change in some observer's knowledge of the state of the system, then such a process seems to require a preferred notion of distant simultaneity - when the wave function collapses, it does so everywhere at once.

E-mail address: wmyrvold@uwo.ca (W.C. Myrvold). 
Many writers have come to the conclusion that state vector collapse is fundamentally at odds with relativity. Cushing (1994, p. 189), for example, writes,

At the level of the state vector, all Lorentz frames are not equivalent, although all predictions for observables are. If one takes the wave function as being merely a mathematical device for calculation, then he need not see a conflict with Lorentz invariance (i.e., with "relativity", loosely speaking). However, if one takes the wave function as representing a physical reality..., then all Lorentz frames are not equivalent at the level of individual processes.

In a similar vein, Maudlin (1996) has suggested that a successful account of EPRBell correlations may require adding a preferred foliation to relativistic spacetime, and that, with respect to this foliation, "the best one could do would be for the wave function to evolve sometimes relativistically and sometimes (during collapse) nonrelativistically" (p. 297).

The purpose of this paper is to examine some arguments that purport to demonstrate the incompatibility of the collapse postulate with relativity theory, and to argue that they do not succeed. Attention to the transformation properties of quantum mechanical states undergoing unitary, non-collapse evolution points the way to a treatment of collapse evolution consistent with the demands of relativity.

As suggested above, the account of quantum-mechanical measurement processes that will be adopted here will be one that takes the "collapse" suggested by von Neumann (1932/1955) to be a real physical process. In spite of some promising work that has been done in the direction of providing a theory of what occurs during a measurement (see, e.g., Percival, 1998; Pearle, 1997, 1999a, and references therein), it is not thought that a fully satisfactory account of the measurement process can yet be provided. Nevertheless, if a measurement does indeed yield a definite result, and if ideal, repeatable measurements yield the same result upon repetition, and if the state vector is a complete description of the quantum-mechanical system, then the state of the system must, as von Neumann realized, end up in an eigenstate of the operator corresponding to the observable measured. I will assume that this is the case.

A few words are in order about what relativity will be taken to require, and what is entailed by a claim of "peaceful coexistence" between a theory and special relativity. ${ }^{1}$ Clearly, Lorentz invariance of predictions regarding experimental outcomes is required. Lorentz invariance at the phenomenal level, however, is not sufficient; the processes that underline the observable phenomena must not pick out a preferred reference frame. Special relativity, is, simply, the theory that the structure of spacetime is that of Minkowski spacetime, whose invariance group is the Poincare group. A theory that introduces additional structure, such as a preferred notion of distant simultaneity, even if it is formulated in a manifestly covariant way, will not, for the purposes of this paper, be regarded as being properly relativistic. This holds even if, according to the theory, in-principle limitations on the accuracy of measurement or on control of certain parameters render it impossible to determine

\footnotetext{
${ }^{1}$ The phrase "peaceful coexistence" was introduced by Shimony (1978).
} 
the preferred hyperplanes of simultaneity or to exploit the nonlocality in the theory for the purposes of superluminal signalling.

There is a tendency, in the literature on relativity, to refer to quantities that are not invariant with respect to reference frame as "observer-dependent". This carries with it the unfortunate suggestion that there is something subjective about such quantities. Where "observer" is not being used as a misleading synonym for "coordinate system" (as it is in Schutz, 1990), the assumption seems to be that every observer is associated with a particular reference frame, presumably the observer's rest frame, and that accompanying every observer is an infinitely extended hyperplane of simultaneity that is that observer's extended present - an assumption that Stein (1991) has cogently criticized. Anyone is free to employ a reference frame and coordinate system of his or her choosing; the lesson of special relativity is that all inertial reference frames can be employed interchangeably.

Unlike Fleming (1989), I will not assume that each detector is associated with a particular foliation of spacetime. I will also refrain from invoking observables that exhibit irreducible dependence on reference frames or on spacelike hyperplanes. In formulating the special theory of relativity, Einstein was able to show that quantities that vary between reference frames - such as the length of an object, or the temporal interval between events - can be regarded as supervening on invariant facts, such as local coincidences of measuring rods and clock-readings. The differing accounts given by differing reference frames, for this reason, can be regarded as nothing more than different descriptions of the same reality. There can therefore be no question as to which of these accounts is correct, as there is no disagreement among them over matters of fact; what differs is only the choice of coordinates used. The goal of this paper is to argue that the differing state histories yielded by different foliations can be regarded also as differing only with respect to the coordinates used-nonlocal observables are definable in terms of local observables, and state vectors, even entangled ones, are superpositions of state vectors that factor into local parts. A theory, such as Fleming's, that introduces irreducibly extended quantities faces an additional burden of reconciling these with the notion that different reference frames merely yield differing accounts of the same processes and events. ${ }^{2}$

The term "quantum mechanics" is usually taken to refer to a quantum theory of systems with a finite number of degrees of freedom-for example, a system consisting of a fixed, finite number of particles. It is contrasted with quantum field theory, which is the quantum theory of systems with an infinite number of degrees of freedom, or, in other words, the quantum theory of fields. There are well-known difficulties associated with an attempt to formulate a relativistic quantum mechanics (e.g., see Malament, 1996 for a lucid presentation of the impossibility of a welldefined relativistic quantum-mechanical position operator), and the consensus seems to be that "there is no acceptable middle ground between ordinary, non-relativistic quantum (particle) mechanics and relativistic quantum field theory" (Malament, 1996, p. 2; for a dissenting view, see Fleming \& Butterfield, 2000). These difficulties

\footnotetext{
${ }^{2}$ In spite of some differences in outlook, it should be stressed that the present paper is indebted to Fleming's $(1989,1996)$ discussions of foliation-relative collapse.
} 
become less acute when the distance scales involved are large compared to the Compton wavelength $\lambda_{C}=\hbar / m_{0} c$ of the system in question, and one can introduce an approximate notion of localization that is valid at such distance scales. The sorts of arguments against peaceful coexistence with which this paper is concerned seem to be independent of these issues. They concern themselves with measurements performed on entangled systems whose separation can be made arbitrarily large, and avail themselves freely of the notion of the locations of the subsystems in question. Aharonov and Albert, who will be the source of two of the arguments to be considered, make this qualification explicitly:

Throughout this work, particles are taken to be localized only within regions larger than the Compton wavelengths; the phenomenon of pair creation then can, to any desired accuracy, be ignored, and we will not need to have recourse to the various mathematical devices (Newton-Wigner operators and the like) which become indispensable to any discussion of the localization of particles to within regions smaller than that. The length scales which are of interest for our present consideration (the separations between the boxes in Sec. I, say [see Example 2, below]) can always be made as large as we like, and we shall take them here to be sufficiently large that, on such scales, the Compton wavelengths of the particles involved can be neglected altogether. On such scales, the full field theory can always accommodate the notion of a single, relativistic, quantum-mechanical particle, such as occupies us in this investigation, and wherein considerations of relativistic covariance, as the reader shall discover, continue to play an essential and problematic role. (1984, p. 233).

In this paper, we will concern ourselves with silver atoms, Stern-Gerlach apparati, and the like, systems that, at least to a high degree of approximation, can be regarded as confined to bounded regions of space, and we will assume that the systems are confined to regions of space that are large compared to the Compton wavelengths of the systems in question but small compared to the distances between them. If Aharonov and Albert's arguments, and the other arguments to be considered here, are successful, they exhibit problems associated with the relativistic covariance of quantum states that would persist when the approximation within which they work is lifted. A defense of the compatibility of quantum theory with special relativity must, therefore, address these arguments.

The conclusions of this paper must therefore be regarded as limited to the scope of the approximation invoked. A thorough treatment, beyond the scope of this paper, of the relations between quantum theory and relativity will have to face foursquare the problems associated with relativistic localization.

\section{Arguments against a covariant account of collapse}

Three examples will be considered to focus attention on the reasons why a relativistic account of collapse is thought by some to be impossible. 


\subsection{Example 1}

The first example to be considered involves the familiar EPR-Bohm thought experiment. A pair of spin- $\frac{1}{2}$ particles is prepared in the singlet state:

$$
\begin{aligned}
|\psi\rangle_{\text {singlet }} & =\frac{1}{\sqrt{2}}\left(|z+\rangle_{1} \otimes|z-\rangle_{2}-|z-\rangle_{1} \otimes|z+\rangle_{2}\right), \\
& =\frac{1}{\sqrt{2}}\left(|x+\rangle_{1} \otimes|x-\rangle_{2}-|x-\rangle_{1} \otimes|x+\rangle_{2}\right) .
\end{aligned}
$$

The particles are permitted to fly apart, and some time after preparation spin measurements are performed, at spacelike separation, on the two particles. Let us suppose that the two measurements are simultaneous with respect to the laboratory frame $K$, and let $A$ and $B$ be the regions in which measurements are performed on particles 1 and 2, respectively. Suppose that a spin- $x$ measurement is performed on particle 1 and a spin- $z$ measurement is performed on particle 2, and suppose that the result is +1 for each measurement. Let $\Sigma$ be a reference frame with respect to which the measurement at $A$ occurs before the measurement at $B$, and let $\Sigma^{\prime}$ be a reference frame with respect to which the temporal order of the two measurements is reversed.

The history of the two-particle system, with respect to $\Sigma$, runs: particle 1 reaches the apparatus at $A$ in an entangled state with particle 2. The measurement of $\sigma_{1 x}$ at $A$ collapses the state to $|x+\rangle_{1} \otimes|x-\rangle_{2}$. Particle 2 , therefore, reaches the apparatus at $B$ disentangled from particle 1 , in the state $|x-\rangle_{2}$. The measurement of $\sigma_{2 z}$ at $B$ projects this state into $|z+\rangle_{2}$.

With respect to $\Sigma^{\prime}$, we have: particle 2 reaches the apparatus at $B$ in an entangled state with particle 1 . The measurement of $\sigma_{2 z}$ at $B$ collapses the state to $|z-\rangle_{1} \otimes|z+\rangle_{2}$. Particle 1, therefore, reaches the apparatus at $A$ disentangled from particle 2 , in the state $|z-\rangle_{1}$. The measurement of $\sigma_{1 x}$ at $A$ projects this state into $|x+\rangle_{1}$.

The two accounts, therefore, disagree as to which measurement collapses the singlet state into a factorizable state. Moreover, on the account according to $\Sigma$, particle 2 arrives at $B$ in the state $|x-\rangle_{2}$, a state which, on the account given by $\Sigma^{\prime}$, it is never in, whereas, according to $\Sigma^{\prime}$, particle 1 arrives at $A$ in the state $|z-\rangle_{1}$, a state which, on the account given by $\Sigma$, it is never in.

If the example is modified so that the same direction of spin is measured on both particles, then the result of the second measurement is predetermined by the result of the first, and hence the two frames will disagree on which measurements are chance events and which have predetermined outcomes. With respect to $\Sigma$, the result of the measurement at $A$ is a chance event, with equal probabilities for each of the two possible outcomes, whereas the measurement at $B$ can have only one possible outcome, namely, a spin opposite to the outcome of the $A$-measurement. The account given by $\Sigma^{\prime}$, on the other hand, has it the other way around: the measurement result at $B$ is a chance event, and the result at $A$ predetermined by the result of the previous measurement at $B$. 
Despite these differences, the probability for any pair of outcomes of any pair of measurements is, as a simple calculation shows, independent of the reference frame used to evaluate it. This circumstance is quite general. In the absence of explicitly non-local interaction terms in the Hamiltonian, probabilities for outcomes of measurements of local observables do not depend on whether a measurement has taken place at spacelike separation; this is the content of the "no-signalling" theorems (Eberhard, 1978, Ghirardi, Rimini, \& Weber, 1980). Thus, so long as the local evolutions are Lorentz invariant (governed, say, by the Dirac equation), we have Lorentz invariance at the phenomenal level. Peaceful coexistence between special relativity and quantum mechanics, realistically construed, requires more; what is needed is "a conceptually coherent reconciliation of the descriptions from the standpoints of $\Sigma$ and $\Sigma^{\prime \prime}$ (Shimony, 1986, p. 195; Shimony, 1993, p. 153). It is just such a reconciliation that Cushing and Maudlin declare to be impossible in the quotations that open this paper.

\subsection{Example 2}

The second example, stemming from Aharonov and Albert (1984), is conceptually similar, except that it involves only a single particle. Initially the particle may be located in one of three boxes, located at $\mathbf{x}_{1}, \mathbf{x}_{2}$, and $\mathbf{x}_{3}$, with equal probability for each box. Boxes 1 and 2 are opened at space-like separation, and the particle is found in neither. Let $\left|\mathbf{x}_{1}\right\rangle$ be a state in which the particle is localized in box $i$, and let $\Sigma$ be a frame with respect to which box 1 is opened first. With respect to this frame, the (unnormalized) initial state of the particle is

$$
|\alpha\rangle=\left|\mathbf{x}_{1}\right\rangle+\left|\mathbf{x}_{2}\right\rangle+\left|\mathbf{x}_{3}\right\rangle \text {. }
$$

After box 1 is opened and before box 2 is opened, the state is

$$
|\beta\rangle=\left|\mathbf{x}_{2}\right\rangle+\left|\mathbf{x}_{3}\right\rangle \text {. }
$$

After box 2 is opened, the state is $\left|\mathbf{x}_{3}\right\rangle$.

Now consider a reference frame, $\Sigma^{\prime}$, with respect to which box 2 is opened first. Let $\left|\mathbf{x}_{i}^{\prime}\right\rangle$ be the Lorentz transform of the state $\left|\mathbf{x}_{i}\right\rangle$. According to $\Sigma^{\prime}$ the intermediate state, between openings, is

$$
|\gamma\rangle=\left|\mathbf{x}_{1}^{\prime}\right\rangle+\left|\mathbf{x}_{3}^{\prime}\right\rangle \text {. }
$$

In connection with this example, Aharonov and Albert (1984, p. 228) remark,

The first observer will judge that the system is for a certain time $\left(t_{1}<t<t_{2}\right)$ in the state $|\beta\rangle$ (more precisely, he will judge that, within that interval, any measurement of $B$, where $B|\beta\rangle=\beta|\beta\rangle$, will with certainty yield $B=\beta$ ); the second will judge that it is never in that state (nor in its Lorentz transform $\left|\beta^{\prime}\right\rangle$ ) but, rather, is for a certain time in the state $|\gamma\rangle$ albeit that these two states and the two histories ... of which they form parts are patently not Lorentz transforms of one another. 


\subsection{Example 3}

The third example to be considered also stems from Aharonov and Albert (1980, 1981). Consider a pair of spin- $\frac{1}{2}$ particles, located at positions $\mathbf{x}_{1}$ and $\mathbf{x}_{2}$, respectively, in the singlet spin state. This spin state of the combined system is characterized uniquely by the conditions,

$$
\left(\sigma_{1 x}+\sigma_{2 x}\right)|\psi\rangle=\left(\sigma_{1 y}+\sigma_{2 y}\right)|\psi\rangle=\left(\sigma_{1 z}+\sigma_{2 z}\right)|\psi\rangle=0 .
$$

More succinctly,

$$
\left(\boldsymbol{\sigma}_{1}+\boldsymbol{\sigma}_{2}\right)|\psi\rangle=\mathbf{0} .
$$

Particle 1 interacts with a measuring device, with commuting pointer observables $\mathbf{q}_{1}=\left\{q_{1 x}, q_{1 y}, q_{1 z}\right\}$, via the interaction Hamiltonian

$$
H_{1}=g_{1}(t) \boldsymbol{\sigma}_{1} \cdot \mathbf{p}_{1} \text {, }
$$

where $\mathbf{p}_{1}=\left\{p_{1 x}, p_{1 y}, p_{1 z}\right\}$ are the momenta conjugate to $\mathbf{q}_{1}$, and $g_{1}(t)$ is a function that is equal to zero before and after the measurement interaction and is non-zero during the measurement interaction. Similarly, particle 2 interacts with a measuring device with pointer observables $\mathbf{q}_{2}$ via the Hamiltonian

$$
H_{2}=-g_{2}(t) \boldsymbol{\sigma}_{2} \cdot \mathbf{p}_{2} \text {. }
$$

The total interaction Hamiltonian is, therefore,

$$
H_{\mathrm{INT}}=H_{1}+H_{2}=g_{1}(t) \boldsymbol{\sigma}_{1} \cdot \mathbf{p}_{1}-g_{2}(t) \boldsymbol{\sigma}_{2} \cdot \mathbf{p}_{2} .
$$

Now suppose that $g_{1}(t)=g_{2}(t)=g(t)$; that is, the two measurement processes are synchronized. Then we have

$$
\begin{aligned}
H_{\mathrm{INT}} & =g(t)\left(\boldsymbol{\sigma}_{1} \cdot \mathbf{p}_{1}-\boldsymbol{\sigma}_{2} \cdot \mathbf{p}_{2}\right) \\
& =\frac{1}{2} g(t)\left[\left(\boldsymbol{\sigma}_{1}+\boldsymbol{\sigma}_{2}\right) \cdot\left(\mathbf{p}_{1}-\mathbf{p}_{2}\right)+\left(\boldsymbol{\sigma}_{1}-\boldsymbol{\sigma}_{2}\right) \cdot\left(\mathbf{p}_{1}+\mathbf{p}_{2}\right)\right] .
\end{aligned}
$$

The measurement devices are prepared initially in a simultaneous eigenstate of $\mathbf{q}_{1}-\mathbf{q}_{2}$ and $\mathbf{p}_{1}+\mathbf{p}_{2}$, each with eigenvalue $\mathbf{0}$. Since $\mathbf{q}_{1}$ and $\mathbf{q}_{2}$ do not commute with $\mathbf{p}_{1}+\mathbf{p}_{2}$, and $\mathbf{p}_{1}$ and $\mathbf{p}_{2}$ do not commute with $\mathbf{q}_{1}-\mathbf{q}_{2}$, this will not be an eigenstate of $\mathbf{q}_{1}, \mathbf{q}_{2}, \mathbf{p}_{1}$, or $\mathbf{p}_{2}$, and will in fact be an entangled state of the two devices. Furthermore, since $\mathbf{p}_{1}-\mathbf{p}_{2}$ does not commute with $\mathbf{q}_{1}-\mathbf{q}_{2}$, the total state of the system will be an eigenstate of the Hamiltonian if and only if $\left(\boldsymbol{\sigma}_{1}+\boldsymbol{\sigma}_{2}\right)|\psi\rangle=\mathbf{0}$ that is, if and only if the spin state of the particle pair is the singlet state.

The Heisenberg-picture time evolution of the pointer observables is given by

$$
\begin{aligned}
\frac{\mathrm{d}}{\mathrm{d} t} \mathbf{q}_{1} & =\frac{1}{\mathrm{i} \hbar}\left[\mathbf{q}_{1}, H_{\mathrm{INT}}\right]=\frac{g(t)}{\mathrm{i} \hbar}\left[\mathbf{q}_{1}, \boldsymbol{\sigma}_{1} \cdot \mathbf{p}_{1}\right], \\
& =g(t) \boldsymbol{\sigma}_{1}, \\
\frac{\mathrm{d}}{\mathrm{d} t} \mathbf{q}_{2} & =\frac{1}{\mathrm{i} \hbar}\left[\mathbf{q}_{2}, H_{\mathrm{INT}}\right]=-\frac{g(t)}{\mathrm{i} \hbar}\left[\mathbf{q}_{2}, \boldsymbol{\sigma}_{2} \cdot \mathbf{p}_{2}\right], \\
& =-g(t) \boldsymbol{\sigma}_{2} .
\end{aligned}
$$


Therefore,

$$
\frac{\mathrm{d}}{\mathrm{d} t}\left(\mathbf{q}_{1}-\mathbf{q}_{2}\right)=g(t)\left(\boldsymbol{\sigma}_{1}+\boldsymbol{\sigma}_{2}\right) .
$$

The measurement devices remain in the eigenstate $\mathbf{q}_{1}-\mathbf{q}_{2}=\mathbf{0}$ if and only if the spin state of the two particles is the singlet state. An observation of $\mathbf{q}_{1}-\mathbf{q}_{2}$ after the measurement interaction is certain to yield the value $\mathbf{0}$ if and only if the spin state of the two particles is the singlet state, and the measurement interaction leaves that state undisturbed. If the initial state is not the singlet state, there is a non-zero probability that an observation of $\mathbf{q}_{1}-\mathbf{q}_{2}$ will yield some value other than zero. Repeating the measurement sufficiently many times can render the probability of getting $\mathbf{q}_{1}-\mathbf{q}_{2} \neq 0$ as high as one would like for any initial state other than the singlet state. If the initial state is the singlet state, therefore, one can verify that it is the singlet state (or close to it) to as high degree of probability as one would like, without disturbing the state. For this reason, Aharonov and Albert refer to the experiment just outlined as a verification of the initial state.

The conclusion that the experiment leaves the system undisturbed depends on the condition $g_{1}(t)=g_{2}(t)=g(t)$, and this is not an invariant relation. If we do not have $g_{1}(t)=g_{2}(t)$, then the system does not remain in the singlet state. To see this, first recall that the measuring devices were prepared in an eigenstate $\mathbf{p}_{1}+\mathbf{p}_{2}=\mathbf{0}$. As the Hamiltonian does not contain the coordinates $\mathbf{q}_{1}, \mathbf{q}_{2}$, this relation holds throughout the motion. On the subspace of states for which $\left(\mathbf{p}_{1}+\mathbf{p}_{2}\right)|\psi\rangle=\mathbf{0}$, the interaction Hamiltonian is equal to

$$
\begin{aligned}
H_{\mathrm{INT}} & =g_{1}(t) \boldsymbol{\sigma}_{1} \cdot \mathbf{p}_{1}-g_{2}(t) \boldsymbol{\sigma}_{2} \cdot \mathbf{p}_{2}, \\
& =\frac{1}{2}\left(g_{1}(t) \boldsymbol{\sigma}_{1}+g_{2}(t) \boldsymbol{\sigma}_{2}\right) \cdot\left(\mathbf{p}_{1}-\mathbf{p}_{2}\right)-\frac{1}{2}\left(g_{1}(t) \boldsymbol{\sigma}_{1}-g_{2}(t) \boldsymbol{\sigma}_{2}\right) \cdot\left(\mathbf{p}_{1}+\mathbf{p}_{2}\right), \\
& =\frac{1}{2}\left(g_{1}(t) \boldsymbol{\sigma}_{1}+g_{2}(t) \boldsymbol{\sigma}_{2}\right) \cdot\left(\mathbf{p}_{1}-\mathbf{p}_{2}\right), \\
& \left.=\frac{1}{4}\left[\left(g_{1}(t)+g_{2}(t)\right)\left(\boldsymbol{\sigma}_{1}+\boldsymbol{\sigma}_{2}\right)+\left(g_{1}(t)-g_{2}(t)\right)\right)\left(\boldsymbol{\sigma}_{1}-\boldsymbol{\sigma}_{2}\right)\right] \cdot\left(\mathbf{p}_{1}-\mathbf{p}_{2}\right) .
\end{aligned}
$$

Therefore, in order for the total state $|\psi\rangle$ of the system to be an eigenstate of the Hamiltonian, it must satisfy

$$
\left[\left(g_{1}(t)+g_{2}(t)\right)\left(\boldsymbol{\sigma}_{1}+\boldsymbol{\sigma}_{2}\right)+\left(g_{1}(t)-g_{2}(t)\right)\left(\boldsymbol{\sigma}_{1}-\boldsymbol{\sigma}_{2}\right)\right]|\psi\rangle=\mathbf{0} .
$$

If the initial spin state is the singlet state, Eq. (14) holds if and only if $g_{1}(t)=g_{2}(t)$ - that is, the measurement is non-disturbing if and only if the two measurement interactions are synchronized. The described experiment, which, with respect to one reference frame, merely verifies that the spin state of the two particles is the singlet state without disturbing it, with respect to any other reference frame first disturbs the state, then restores it to the singlet state.

In connection with an example similar to the one just discussed, Aharonov and Albert (1984, p. 229) remark,

All of this begins to suggest something curious about the covariance of the state vector. A measurement which is judged by an observer in $K$ to verify [the 
state] without disturbing the system will necessarily disturb the system, as judged by an observer in $K^{\prime}$. The measuring process, so far as $K$ is concerned, disrupts (as it were) the transformation properties of the state and disrupts its covariance, without in any way disrupting the history of the state itself.

It is the goal of the next section to show that such processes do not, in fact, interfere with the transformation properties of quantum states, if proper attention is paid to the manner in which evolving states of spatially extended systems must be transformed. This will provide a clue to understanding how the state histories in the first two examples can, in fact, be descriptions, with respect to different reference frames, of the same temporal evolution of the systems involved.

\section{Quantum states and their Lorentz transforms}

Let us first consider two systems, $S_{1}$ and $S_{2}$, that, during the time of interest, are each confined to small regions $A$ and $B$, located at time $t$ (with respect to reference frame $\Sigma$ ) at $\mathbf{x}_{1}(t)$ and $\mathbf{x}_{2}(t)$, respectively. Assume that $A$ and $B$ are small enough, compared to the distance between them, that they may be treated as if they were points, but large enough that the systems may indeed be regarded as confined to the regions; that is, the dimensions of $A$ and $B$ are large compared to the Compton wavelengths of the systems located therein. Let $\mathscr{H}_{1}$ and $\mathscr{H}_{2}$ be the Hilbert spaces corresponding to the internal degrees of freedom of $S_{1}$ and $S_{2}$, respectively; the Hilbert space belonging to the joint system $S_{1}+S_{2}$ is thus the tensor product space $\mathscr{H}_{1} \otimes \mathscr{H}_{2}$.

Consider first the case in which the two systems are not entangled. If the state of $S_{1}$ at time $t$ is given by $|u(t)\rangle \in \mathscr{H}_{1}$ and the state of $S_{2}$ is $|v(t)\rangle \in \mathscr{H}_{2}$, then the state of $S_{1}+S_{2}$ at time $t$ is

$$
|\psi(t)\rangle_{12}=|u(t)\rangle_{1} \otimes|v(t)\rangle_{2} .
$$

Consider a Lorentz boost

$$
\begin{aligned}
& x^{\prime}=\gamma(x-v t), \\
& t^{\prime}=\gamma\left(t-\frac{v x}{c^{2}}\right),
\end{aligned}
$$

where $\gamma / \sqrt{1-v^{2} / c^{2}}$. By Wigner's theorem (see Weinberg 1995, pp. 91-96), there is a unitary transformation operator $\Lambda_{1}$ that takes vectors in $\mathscr{H}_{1}$ into their transforms under the Lorentz boost (16). Similarly, there is a unitary Lorentz boost operator $\Lambda_{2}$ on $\mathscr{H}_{2}$. As Dickson and Clifton (1998, p. 15) have shown, the transformation operator on $\mathscr{H}_{1} \otimes \mathscr{H}_{2}$ is simply $\Lambda_{1} \otimes \Lambda_{2}$. Therefore, for any time $t$, the Lorentz transform of $|\psi(t)\rangle_{12}$ is

$$
\begin{aligned}
\left|\psi^{\prime}\right\rangle_{12} & =\Lambda_{1}|u(t)\rangle_{1} \otimes \Lambda_{2}|v(t)\rangle_{2}, \\
& =\left|u^{\prime}\left(t_{1}^{\prime}(t)\right)\right\rangle_{1} \otimes\left|v^{\prime}\left(t_{2}^{\prime}(t)\right)\right\rangle_{2},
\end{aligned}
$$


where

$$
\begin{aligned}
& t_{1}^{\prime}(t)=\gamma\left(t-\frac{v x_{1}(t)}{c^{2}}\right), \\
& t_{2}^{\prime}(t)=\gamma\left(t-\frac{v x_{2}(t)}{c^{2}}\right) .
\end{aligned}
$$

That is: to say that, as seen in reference frame $\Sigma$, the state at time $t$ is $|u\rangle \otimes|v\rangle$, is to say that, as seen by reference frame $\Sigma^{\prime}, S_{1}$ is in state $\left|u^{\prime}\right\rangle$ at time $t_{1}^{\prime}(t)$, and that $S_{2}$ is in state $\left|v^{\prime}\right\rangle$ at time $t_{2}^{\prime}(t)$. Statements about the instantaneous state of the system, with respect to $\Sigma$, go over into statements about the states of the parts of the system at two different times with respect to $\Sigma^{\prime}$. Instantaneous states of $S_{1}+S_{2}$, with respect to $\Sigma^{\prime}$ are of the form

$$
\begin{aligned}
\left|\psi^{\prime}\left(t^{\prime}\right)\right\rangle_{12} & =\left|u^{\prime}\left(t^{\prime}\right)\right\rangle \otimes\left|v^{\prime}\left(t^{\prime}\right)\right\rangle, \\
& =\Lambda_{1}\left|u\left(t_{1}\left(t^{\prime}\right)\right)\right\rangle \otimes \Lambda_{2}\left|v\left(t_{2}\left(t^{\prime}\right)\right)\right\rangle,
\end{aligned}
$$

where

$$
\begin{aligned}
& t_{1}\left(t^{\prime}\right)=\gamma\left(t^{\prime}+\frac{v x_{1}^{\prime}\left(t^{\prime}\right)}{c^{2}}\right), \\
& t_{2}\left(t^{\prime}\right)=\gamma\left(t^{\prime}+\frac{v x_{2}^{\prime}\left(t^{\prime}\right)}{c^{2}}\right) .
\end{aligned}
$$

The instantaneous state of an extended quantum system is, therefore, defined only relative to a spacelike hyperplane (or, more generally, a spacelike hypersurface) of simultaneity. This is not a radically new postulate, but a simple consequence of the notion 'state at a time', plus the relativity of simultaneity. A state history of an extended system is defined only with respect to a choice of a foliation of spacetime into spacelike hypersurfaces. This was pointed out by Dirac (1933), and was elaborated by Tomonaga (1946) and Schwinger (1948). If one works within the Heisenberg picture, in which it is operators corresponding to fields that evolve, rather than states, and concerns oneself with operators corresponding to local observables, then one need not deal with foliation-dependent states (though field quantities defined in terms of spatial integrals of local fields are, on such a picture, foliation-relative). The language of foliation-relative state evolution is the translation of this Heisenberg-picture evolution back into the Schrödinger picture.

The state of a system can remain constant for all time with respect to one foliation while not remaining constant with respect to another. Suppose, for example, that our system consists of two spin- $\frac{1}{2}$ particles, with charge $e$, initially in the state $|z+\rangle_{1} \otimes|z-\rangle_{2}$, at rest with respect to $\Sigma$, and that at a certain time $\tau$ a magnetic field in the $\hat{z}$-direction, of strength $\mathscr{B}$, is switched on. After time $\tau$, the Hamiltonian 
of the system (with degrees of freedom other than spin neglected) is

$$
\begin{aligned}
H & =-\frac{e \hbar \mathscr{B}}{2 m c}\left(\sigma_{1 z} \otimes I_{2}+I_{1} \otimes \sigma_{2 z}\right), \\
& =-\hbar \omega\left(\sigma_{1 z} \otimes I_{2}+I_{1} \otimes \sigma_{2 z}\right),
\end{aligned}
$$

where $\omega=e \mathscr{B} / 2 m c$. The subsequent evolution of the system is, therefore,

$$
\begin{aligned}
U(t)|z+\rangle_{1} \otimes|z-\rangle_{2} & =\mathrm{e}^{(H(t-\tau)) /-i \hbar}|z+\rangle_{1} \otimes|z-\rangle_{2}, \\
& =\mathrm{e}^{\mathrm{i} \omega(t-\tau)}|z+\rangle_{1} \otimes \mathrm{e}^{-\mathrm{i} \omega(t-\tau)}|z-\rangle_{2}, \\
& =|z+\rangle_{1} \otimes|z-\rangle_{2} .
\end{aligned}
$$

The state remains unchanged for all time only if the magnetic field at $A$ is switched at the same time as the magnetic field at $B$. If the two magnetic fields are applied at distinct times $\tau_{1}$ and $\tau_{2}$, we have, after both fields are switched on,

$$
\begin{aligned}
U(t)|z+\rangle_{1} \otimes|z-\rangle_{2} & =\mathrm{e}^{\mathrm{i} \omega\left(t-\tau_{1}\right)}|z+\rangle_{1} \otimes \mathrm{e}^{-\mathrm{i} \omega\left(t-\tau_{2}\right)}|z-\rangle_{2}, \\
& =\mathrm{e}^{\mathrm{i} \omega\left(\tau_{2}-\tau_{1}\right)}|z+\rangle_{1} \otimes|z-\rangle_{2} .
\end{aligned}
$$

In this example, two reference frames, one in which the fields are switched on simultaneously and one in which they are not, will represent the state of the system differently, but the two accounts will differ only by a multiplicative factor of magnitude one. Consider, however, the situation in which the initial state of the particle pair is the singlet state. Then we have, after both fields are switched on,

$$
\begin{aligned}
|\psi(t)\rangle= & U(t)|\psi\rangle_{\text {singlet }}, \\
= & \frac{1}{\sqrt{2}}\left(\mathrm{e}^{-\mathrm{i} \omega\left(t-\tau_{1}\right)}|z+\rangle_{1} \otimes \mathrm{e}^{-\mathrm{i} \omega\left(t-\tau_{2}\right)}|z-\rangle_{2}\right. \\
& \left.-\mathrm{e}^{\mathrm{i} \omega\left(t-\tau_{1}\right)}|z-\rangle_{1} \otimes \mathrm{e}^{\mathrm{i} \omega\left(t-\tau_{2}\right)}|z+\rangle_{2}\right), \\
= & \frac{1}{\sqrt{2}}\left(\mathrm{e}^{\mathrm{i} \omega\left(\tau_{2}-\tau_{1}\right)}|z+\rangle_{1} \otimes|z-\rangle_{2}-\mathrm{e}^{-\mathrm{i} \omega\left(\tau_{2}-\tau_{1}\right)}|z-\rangle_{1} \otimes|z+\rangle_{2}\right) .
\end{aligned}
$$

Unless $\tau_{1}=\tau_{2}$-that is, unless the switching on of the magnetic fields occurs simultaneously at $A$ and $B$ - this is not the singlet state. For example, for a system in state (24), a measurement of spin- $x$ on both particles is not guaranteed to yield opposite results; there is a probability equal to $\sin ^{2}\left(\omega\left(\tau_{2}-\tau_{1}\right)\right)$ that the results of the two spin measurements will match.

It may seem, therefore, that there is a conflict between the probabilities of measurement results calculated with respect to a reference frame $\Sigma$, in which the switching events are simultaneous, and a frame $\Sigma^{\prime}$, in which they are not simultaneous. According to $\Sigma$, a measurement of spin- $x$ on both particles is guaranteed to yield opposite results, whereas, according to $\Sigma^{\prime}$, a measurement of 
spin- $x$ on both particles has a non-zero probability of yielding matching results. The reason that no conflict exists is that these probabilities are probabilities of the results of simultaneous measurements on the two particles. Both frames agree that measurements of spin- $x$ on both particles that are simultaneous with respect to $\Sigma$ will always yield opposite results, and that measurements of spin- $x$ that are simultaneous with respect to $\Sigma^{\prime}$ have a non-zero probability of yielding matching results.

The circumstance that Aharonov and Albert draw attention to, that the state of a combined system may remain unchanged with respect to one reference frame but not with respect to another, does not depend on the interaction being a measurement interaction, and can obtain in circumstances not regarded as interactions with measuring devices. This may seem to lend support to Aharonov and Albert's suggestion that there is something curious about the covariance of the state vector. Consider the example just discussed. In the absence of the magnetic fields, the system remains in the singlet state with respect to any reference frame. In the presence of the magnetic fields, the system remains in the singlet state when described by a frame in which the switching events are simultaneous, and does not remain in the singlet state when described by a frame in which the switching events are not simultaneous. This seems to require that the same state have two distinct Lorentz transforms!

That this is not the case can be seen if one takes care to include the time evolution of the states in the transformation. Let $U(t)=U_{1}(t) \otimes U_{2}(t)$ be the evolution operator for a composite system consisting of two subsystems located, as above, at $\mathbf{x}_{1}(t)$ and $\mathbf{x}_{2}(t)$, respectively. Let the initial state of the system at time $t=0$, with respect to $\Sigma$, be given by

$$
|\psi(0)\rangle=\sum_{k} c_{k}\left|u_{k}\right\rangle \otimes\left|v_{k}\right\rangle
$$

Then, at time $t$, the state of the system is

$$
U(t)|\psi(0)\rangle=\sum_{k} c_{k} U_{1}(t)\left|u_{k}\right\rangle \otimes U_{2}(t)\left|v_{k}\right\rangle .
$$

The Lorentz transform of the state at time $t$ is

$$
\begin{aligned}
\Lambda U(t)|\psi(0)\rangle & =\left(\Lambda_{1} \otimes \Lambda_{2}\right) U(t)|\psi(0)\rangle, \\
& =\sum_{k} c_{k} \Lambda_{1} U_{1}(t)\left|u_{k}\right\rangle \otimes \Lambda_{2} U_{2}(t)\left|v_{k}\right\rangle .
\end{aligned}
$$

The instantaneous state of the system, with respect to $\Sigma^{\prime}$, at time $t^{\prime}$, is given by

$$
\begin{aligned}
\left|\psi^{\prime}\left(t^{\prime}\right)\right\rangle & =\sum_{k} c_{k} \Lambda_{1} U_{1}\left(t_{1}\left(t^{\prime}\right)\right)\left|u_{k}\right\rangle \otimes \Lambda_{2} U_{2}\left(t_{2}\left(t^{\prime}\right)\right)\left|v_{k}\right\rangle \\
& =\Lambda_{1} U_{1}\left(t_{1}\left(t^{\prime}\right)\right) \otimes \Lambda_{2} U_{2}\left(t_{2}\left(t^{\prime}\right)\right)|\psi(0)\rangle .
\end{aligned}
$$

That is, the transformation from a state, defined with respect to $\Sigma$, along one of $\Sigma$ 's hyperplanes of simultaneity, to a state defined with respect to $\Sigma^{\prime}$, along one of $\Sigma^{\prime}$ 's hyperplanes of simultaneity, involves a combination of Lorentz transformation 
and time-evolution from one hyperplane to another. In our example, this yields

$$
\begin{aligned}
\left|\psi^{\prime}\left(t^{\prime}\right)\right\rangle= & \frac{1}{\sqrt{2}}\left(\mathrm{e}^{\mathrm{i} \omega^{\prime}\left(t^{\prime}-\tau_{1}^{\prime}\right)}\left|z^{\prime}+\right\rangle_{1} \otimes \mathrm{e}^{-\mathrm{i} \omega^{\prime}\left(t^{\prime}-\tau_{2}^{\prime}\right)}\left|z^{\prime}-\right\rangle_{2}\right. \\
& \left.+\mathrm{e}^{-\mathrm{i} \omega^{\prime}\left(t^{\prime}-\tau_{1}^{\prime}\right)}\left|z^{\prime}-\right\rangle_{1} \otimes \mathrm{e}^{\mathrm{i} \omega^{\prime}\left(t^{\prime}-\tau_{2}^{\prime}\right)}\left|z^{\prime}+\right\rangle_{2}\right), \\
= & \frac{1}{\sqrt{2}}\left(\mathrm{e}^{\mathrm{i} \omega^{\prime}\left(\tau_{2}^{\prime}-\tau_{1}^{\prime}\right)}\left|z^{\prime}+\right\rangle_{1} \otimes\left|z^{\prime}-\right\rangle_{2}-\mathrm{e}^{-\mathrm{i} \omega^{\prime}\left(\tau_{2}^{\prime}-\tau_{1}^{\prime}\right)}\left|z^{\prime}-\right\rangle_{1} \otimes\left|z^{\prime}+\right\rangle_{2}\right) .
\end{aligned}
$$

The system remains in the singlet state with respect to $\Sigma$ whether or not the magnetic fields are turned on. In the absence of the magnetic fields, the state is also a singlet state with respect to $\Sigma^{\prime}$. In the presence of the magnetic fields, it would be incorrect to transform the state into a singlet state with respect to $\Sigma^{\prime}$, as such a transformation does not respect the dynamical evolution of the component subsystems.

Transformation (28) assumes that the time evolution operator $U(t)$ is the product of local evolution operators:

$$
U(t)=U_{1}(t) \otimes U_{2}(t)
$$

This will be the case if and only if the system Hamiltonian is a sum of local Hamiltonians:

$$
H=H_{1} \otimes I_{2}+I_{1} \otimes H_{2} .
$$

If the Hamiltonian contained a non-local interaction term, this term would create a need for a preferred notion of distant simultaneity.

Now consider a case in which the magnetic field is applied at time $\tau$ to particle 2 only. This gives, for $t \geqslant \tau$,

$$
|\psi(t)\rangle \frac{1}{\sqrt{2}}\left(\mathrm{e}^{-\mathrm{i} \omega(t-\tau)}|z+\rangle_{1} \otimes|z-\rangle_{2}-\mathrm{e}^{\mathrm{i} \omega(t-\tau)}|z-\rangle_{1} \otimes|z+\rangle_{2}\right) .
$$

Note that this is the same state that would result from a magnetic field of the same strength in the opposite direction applied to particle 1 - it is only the difference between the applied field at $A$ and the applied field at $B$ that appears in the state (32). Nevertheless, there is a matter of fact about whether a field was applied to particle 1 or particle 2, a matter of fact that could be revealed by the behaviour of other particles in the vicinity of particles 1 and 2 . It seems reasonable to consider the evolving state (32) to involve a change pertaining to particle 2 . We should not, however, describe this as a change in particle 2's spin state, as the state does not ascribe the individual particles their own spin states. It is, however, a change pertaining to particle 2's Hilbert space, as is shown by the fact that the evolution operator has the form

$$
U(t)=I_{1} \otimes U_{2}(t) .
$$

In what follows, I will describe such changes - changes that, when the dynamics is taken into account, are properly represented by operators of this sort-as local changes, even though they need not be regarded as changes in intrinsic properties possessed by one or other of the localized subsystems. 
Let us now add collapse to the picture. ${ }^{3}$ It will be helpful to begin with a particular example, and then generalize, and, again, it will helpful to consider first factorizable states and then entangled states as superpositions of factorizable states. Consider, then, two spin- $\frac{1}{2}$ particles, located some distance apart, in regions $A$ and $B$, respectively, and take the initial state to be $|z+\rangle_{1} \otimes|z-\rangle_{2}$. Suppose that a measurement of $\operatorname{spin}-x$ is performed on particle 2. After the measurement, the state of the particle pair becomes one of $|z+\rangle_{1} \otimes|x+\rangle_{2}$ or $|z+\rangle_{1} \otimes|x-\rangle_{2}$, depending on the outcome of the measurement. Suppose that the outcome is +1 , and that the new state is therefore $|z+\rangle_{1} \otimes|x+\rangle_{2}$. Let $P$ be a point on particle 1's worldline that is spacelike separated from the measurement process. There will be reference frames that have it that the measurement on particle 2 is in the future as of $P$, and reference frames according to which the measurement on particle 2 has already occurred as of $P$. More precisely, there will be some reference frame $\Sigma$ such that the hyperplane of simultaneity with respect to $\Sigma$ that passes through $P$ passes through particle 2's worldline to the past of the measurement (call this hyperplane $\sigma_{P}$ ), and there will be some reference frame $\Sigma^{\prime}$ such that the hyperplane of simultaneity with respect to $\Sigma^{\prime}$ passing through $P$ passes through particle 2's wordline to the future of the measurement (call this hyperplane $\sigma_{P}^{\prime}$ ). The instantaneous state defined along $\sigma_{P}$ is the original state $|z+\rangle_{1} \otimes|z-\rangle_{2}$, whereas the state defined along $\sigma_{P}^{\prime}$ is $\left|z^{\prime}+\right\rangle_{1} \otimes\left|x^{\prime}+\right\rangle_{2}$. There is, of course, nothing paradoxical about this; the differing state descriptions are merely consequences of the relativity of distant simultaneity. The change in the state vector is a local one confined to the region $B$ in which the measurement takes place, and affects the global state vector only via its dependence on the part pertaining to $B$.

Call the scenario just described Scenario I, and consider a second scenario in which the initial state is $|z-\rangle_{1} \otimes|z+\rangle_{2}$, spin- $x$ is measured on Particle 2 , and the outcome is again +1 , and call this Scenario II. On Scenario II, the state defined on $\sigma_{P}$ is $|z-\rangle_{1} \otimes|z+\rangle_{2}$, and the state defined on $\sigma_{P}^{\prime}$ is $\left|z^{\prime}-\right\rangle_{1} \otimes\left|x^{\prime}+\right\rangle_{2}$.

Now consider a third scenario, Scenario I + II, which is the superposition of the first two. In this scenario, the initial state is a superposition of the initial states in Scenarios I and II:

$$
|\psi(0)\rangle=\alpha|z+\rangle_{1} \otimes|z-\rangle_{2}+\beta|z-\rangle_{1} \otimes|z+\rangle_{2} .
$$

\footnotetext{
${ }^{3}$ Though, as was mentioned above, the notion of foliation-relative state evolution was present from the very beginning of work on relativistic quantum theories, the application of this notion to state-vector collapse was perhaps first made by Aharonov and Albert (1984). Fleming (1986, 1989) has done more than anyone else to bring the idea of foliation-relative state evolution, and in particular foliation-relative collapse, to the attention of the philosophical community. Maudlin (1994, p. 208) also cites Giovannini (1983) and Dieks (1985) in connection with foliation-dependent collapse theories. Giovannini, however, regards the wave function as a representation of the knowledge some observer has of a system, and wave packet reduction, not as a physical process, but as a reflection of information gained by the observer. (Giovannini associates with each observer a corresponding foliation of spacetime, thereby making observer-relative changes in knowledge into foliation-relative state-vector reductions.) Dieks (1985) works within a 'no-collapse' interpretation in which the state vector evolves unitarily at all times but environmentally induced decoherence leads to what is regarded as an effective collapse.
} 
Again, suppose that a measurement of $\operatorname{spin}-x$ is made on particle 2, and that the result is +1 . The state of the system on this scenario is, at all times (and along any hyperplane) just a superposition of the states in Scenarios I and II. Along $\sigma_{P}$, the state is

$$
\alpha|z+\rangle_{1} \otimes|z-\rangle_{2}+\beta|z-\rangle_{1} \otimes|z+\rangle_{2}
$$

The (unnormalized) state defined on $\sigma_{P}^{\prime}$ is

$$
\begin{aligned}
\alpha \mid z^{\prime} & +\rangle_{1} \otimes\left({ }_{2}\left\langle x^{\prime}+\mid z^{\prime}-\right\rangle_{2}\right)\left|x^{\prime}+\right\rangle_{2}+\beta\left|z^{\prime}-\right\rangle_{1} \otimes\left({ }_{2}\left\langle x^{\prime}+\mid z^{\prime}+\right\rangle_{2}\right)\left|x^{\prime}+\right\rangle_{2}, \\
& =\left(\alpha_{2}\left\langle x^{\prime}+\mid z^{\prime}-\right\rangle_{2}\left|z^{\prime}+\right\rangle_{1}+\beta_{2}\left\langle x^{\prime}+\mid z^{\prime}+\right\rangle_{2}\left|z^{\prime}-\right\rangle_{1}\right) \otimes\left|x^{\prime}+\right\rangle_{2} \\
& =\frac{1}{\sqrt{2}}\left(\alpha\left|z^{\prime}+\right\rangle_{1}+\beta\left|z^{\prime}-\right\rangle_{1}\right) \otimes\left|x^{\prime}+\right\rangle_{2} .
\end{aligned}
$$

Although an entangled state of the two-particle system does not factor into local parts, any state vector can be written as a superposition of terms that do so factor. In the case of unitary evolution, when an operation is performed on one particle (say, particle 2), and not the other, the effect of this operation can be represented as a local change, affecting the global state vector only via its effect on particle 2's state in each term of the superposition. It is because these operations can be represented as local changes, and combined operations on both particles can be represented as combinations of local changes, that such operations do not disrupt the covariance of the state vector, even in the seemingly paradoxical cases in which the state history is an unchanging one along one foliation but not along other foliations.

Something similar happens in the collapse case also. In Scenario I + II, just as in Scenarios I and II, the transition that occurs when a measurement is performed on particle 2 can, for each term of the superposition, be represented as effecting a change in the global state vector only via the effect on the particle 2's state in each term of the superposition. This suggests that we regard such changes as local changes also.

There is, however, an important difference between Scenarios I and II and Scenario I + II. In the first two scenarios, the state of the particle pair is a factorizable state both before and after collapse; the state is a factorizable state on both $\sigma_{P}$ and $\sigma_{P}^{\prime}$. In Scenario I + II, however, the state of the combined system is entangled before the measurement and factorizable afterwards. The state defined on $\sigma_{P}$ is entangled, whereas the state defined on $\sigma_{P}^{\prime}$ is factorizable, even though the two hyperplanes intersect Particle 1's worldline at the same point $P$. This circumstance, a consequence jointly of the relativity of simultaneity and of modelling collapse as a local change in the state vector, can with justice be called the relativity of entanglement. ${ }^{4}$

\footnotetext{
${ }^{4}$ The relativity of entanglement is referred to by Ghirardi and Pearle (1990) as ambiguity about properties. As Rob Clifton (personal communication) has pointed out, the argument of Clifton, Pagonis, and Pitowsky (1992) can be seen as an argument for the relativity of entanglement.
} 
A state-vector collapse, even when affected by a measurement performed on particle 2, affects properties that one is inclined to regard as intrinsic to particle 1 -notably, the probabilities pertaining to subsequent measurements on particle 1 . This is an important disanalogy between collapse and unitary evolution. Whether this means that collapse events cannot be regarded as 'local' depends on whether such properties ought to be regarded as local intrinsic properties of the systems in question; there can be no objection to global properties being affected by local changes pertaining to particle 2. It is the lesson of Bell's theorem that quantummechanical probabilities are not to be regarded as local quantities (we will return to this issue in Sections 4 and 5). By the same token, the reduced density matrices obtained for each subsystem in an entangled state by tracing out the degrees of freedom of the other systems also ought not to be regarded as representing intrinsic states of the component subsystems.

It will be shown below that collapse evolution can be modelled so as not to pick out a preferred foliation, and in such a way that a complete state history given with respect to one foliation uniquely determines the state history with respect to any other foliation (that is, it will be shown that it can be so modelled within the scope of the approximations discussed in Section 1). This lends support to the notion that changes in the global state induced by collapses ought to be regarded as 'local' changes, and this is the terminology that will be adopted below. It should be borne in mind that 'local' is being used here in the sense (appropriate to both unitary and collapse evolution) of: affecting the global state vector via changes in each term of the superposition pertaining to the system on which the measurement is performed, and not in the sense of changes of a local, complete, intrinsic state of some system.

The collapse of the state vector cannot be the result of a linear unitary evolution. We will model a collapse event by a one-parameter family of linear, non-unitary evolution operators, $E(t)$. Consider, for example, a spin- $\frac{1}{2}$ particle, initially in state $|\psi\rangle$, on which a measurement of $\operatorname{spin}-x$ is performed. Schrödinger evolution is represented by a one-parameter family of unitary operators $U(t)$. This evolution is deterministic in the sense that $U(t)$ is determined, for all $t$, by the Hamiltonian of the system. Collapse evolution will be represented by a one-parameter family of operators $E(t)$ such that, for all times $t$ before the measurement $E(t)=I$, and for times $t$ after the measurement $E(t)$ becomes either $P^{|x+\rangle}$ or $P^{|x-\rangle}$, with probability $\left\langle\psi\left|P^{|x+\rangle}\right| \psi\right\rangle /\|\psi\|^{2}$ and $\left\langle\psi\left|P^{|x-\rangle}\right| \psi\right\rangle /\|\psi\|^{2}$, respectively. ${ }^{5}$ (Here $P|\phi\rangle$ is the projection operator onto the subspace spanned by $|\phi\rangle$, i.e., $P^{|\phi\rangle}=|\phi\rangle\langle\phi|/ \|| \phi\rangle \|^{2}$.) The family $E(t)$ is thus not determined by the dynamics of the system but contains a stochastic element-what one has is a one-parameter family of operator-valued

\footnotetext{
${ }^{5}$ The collapse evolution operator, so defined, is not norm-preserving. This is all right so long as we remember to divide by the squared norm of the state in calculating probabilities. The paradoxical-seeming fact that one can represent collapse via linear operators if one is willing to sacrifice unitarity was pointed out by Pearle (1989). On some collapse theories, of which CSL is one, the state only approaches asymptotically a state of complete collapse and at any finite time contains 'tails'. On such a theory, the projection operators here introduced must be regarded as approximations to a more complicated representation of the collapsed state.
} 
random variables, that takes on, for any $t$ some value $E(t)$, with probabilities determined by the details of our collapse theory.

More generally, a collapse will be modelled by a one-parameter family of operators $E(t)$ such that $E(t)$ is equal to $I$ before the collapse, and to $P^{\left|\psi^{\prime}\right\rangle}$, where $\left|\psi^{\prime}\right\rangle$ is whatever state the system happens to collapse to. If two systems $S_{1}$ and $S_{2}$ are spacelike separated during a certain time interval, it will be assumed that during this interval the evolution operator factors:

$$
E(t)=E_{1}(t) \otimes E_{2}(t) .
$$

We now have a coherent meshing of the accounts given by the two reference frames $\Sigma$ and $\Sigma^{\prime}$, in Example 1. With respect to $\Sigma$, the evolution of the system is given by

$$
|\psi(t)\rangle=E_{1}(t) \otimes E_{2}(t)|\psi\rangle_{\text {singlet }},
$$

where

$$
\begin{aligned}
& E_{1}(t)= \begin{cases}I_{1} & \text { before the measurement at } A, \\
P^{|x+\rangle_{1}} & \text { after the measurement at } A .\end{cases} \\
& E_{2}(t)= \begin{cases}I_{2} & \text { before the measurement at } B, \\
P^{|z+\rangle_{2}} & \text { after the measurement at } B .\end{cases}
\end{aligned}
$$

With respect to $\Sigma^{\prime}$, the evolution of the system is given by

$$
\left|\psi^{\prime}\left(t^{\prime}\right)\right\rangle=E_{1}^{\prime}\left(t^{\prime}\right) \otimes E_{2}^{\prime}\left(t^{\prime}\right)\left|\psi^{\prime}\right\rangle_{\text {singlet }},
$$

where

$$
\begin{aligned}
& E_{1}^{\prime}\left(t^{\prime}\right)= \begin{cases}I_{1} & \text { before the measurement at } A, \\
P^{\left|x^{\prime}+\right\rangle_{1}} & \text { after the measurement at } A .\end{cases} \\
& E_{2}^{\prime}\left(t^{\prime}\right)= \begin{cases}I_{2} & \text { before the measurement at } B, \\
P^{\left|z^{\prime}+\right\rangle_{2}} & \text { after the measurement at } B .\end{cases}
\end{aligned}
$$

These operators are simply the Lorentz transforms of the operators (39):

$$
E_{i}^{\prime}\left(t^{\prime}\right)=\Lambda_{i} E_{i}\left(t_{i}\left(t^{\prime}\right)\right) \Lambda_{i}^{\dagger}
$$

Now, let $t_{\alpha}$ be a $\Sigma$ time-coordinate, such that the measurement at $A$ occurs after $t_{\alpha^{-}}$ that is, the hyperplane $t=t_{\alpha}$ lies in the past of the $A$-measurement (see Fig. 1). Let $t_{\beta}$ be a time after the $A$-measurement but before the $B$-measurement, and let $t_{\gamma}$ be a time after both measurements. The account of the evolution of the system, with respect to $\Sigma$, runs: prior to the measurement at $A$, the state of the system is the singlet state: ${ }^{6}$

$$
|\psi(0)\rangle=|x+\rangle_{1} \otimes|x-\rangle_{2}-|x-\rangle_{1} \otimes|x+\rangle_{2}=E_{1}\left(t_{\alpha}\right) \otimes E_{2}\left(t_{\alpha}\right)|\psi(0)\rangle .
$$

\footnotetext{
${ }^{6}$ Since we are discussing a non-unitary collapse evolution in this section, it will be more convenient not to require the state vector to be normalized.
} 


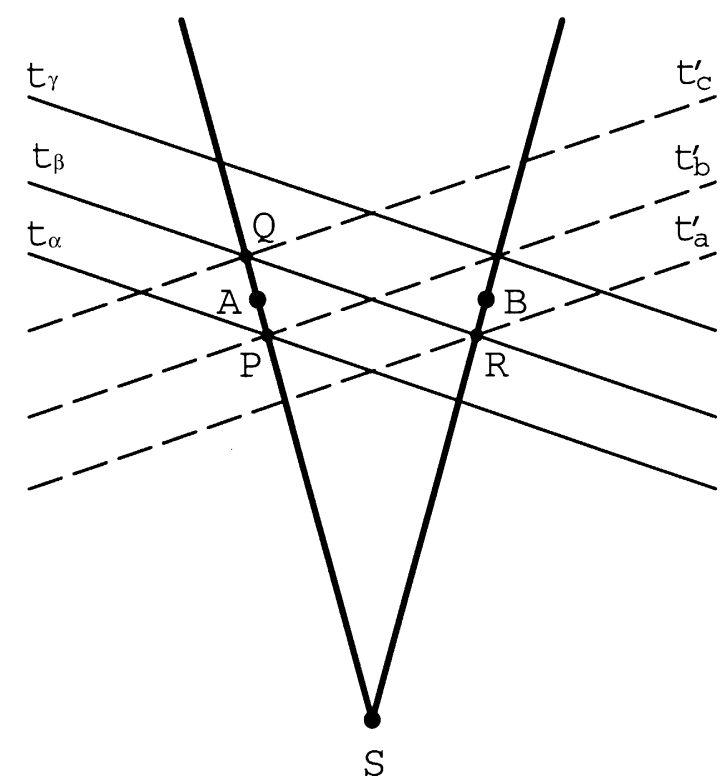

Fig. 1. Spacetime diagram of the EPR-Bohm experiment.

After the $A$-measurement, the state becomes

$$
\begin{aligned}
E_{1}\left(t_{\beta}\right) \otimes E_{2}\left(t_{\beta}\right)|\psi(0)\rangle & =\left(P^{|x+\rangle_{1}} \otimes I_{2}\right)|\psi(0)\rangle, \\
& =P^{|x+\rangle_{1}}|x+\rangle_{1} \otimes|x-\rangle_{2}-P^{|x+\rangle_{1}}|x-\rangle_{1} \otimes|x+\rangle_{2}, \\
& =|x+\rangle_{1} \otimes|x-\rangle_{2} .
\end{aligned}
$$

After the $B$-measurement, the state is

$$
\begin{aligned}
E_{1}\left(t_{\gamma}\right) \otimes E_{2}\left(t_{\gamma}\right)|\psi(0)\rangle & =\left(P^{|x+\rangle_{1}} \otimes P^{|z+\rangle_{2}}\right)|\psi(0)\rangle, \\
& =-\frac{1}{\sqrt{2}}|x+\rangle_{1} \otimes|z+\rangle_{2}
\end{aligned}
$$

Let $t_{\mathrm{a}}^{\prime}, t_{\mathrm{b}}^{\prime}$, and $t_{\mathrm{c}}^{\prime}$ be $\Sigma^{\prime}$ times such that $t_{\mathrm{a}}^{\prime}$ is before the $B$-measurement, $t_{\mathrm{b}}^{\prime}$ is between the two measurements, and $t_{\mathrm{c}}^{\prime}$ is after both measurements. The statehistory with respect to $\Sigma^{\prime}$ runs: before the measurement at $B$, the state of the system is

$$
\left|\psi^{\prime}(0)\right\rangle=\left|z^{\prime}+\right\rangle_{1} \otimes\left|z^{\prime}-\right\rangle_{2}-\left|z^{\prime}-\right\rangle_{1} \otimes\left|z^{\prime}+\right\rangle_{2}=E_{1}^{\prime}\left(t_{\mathrm{a}}^{\prime}\right) \otimes E_{2}^{\prime}\left(t_{\mathrm{a}}^{\prime}\right)\left|\psi^{\prime}(0)\right\rangle .
$$


After the $B$-measurement but before the measurement at $A$, the state is

$$
\begin{aligned}
E_{1}^{\prime}\left(t_{\mathrm{b}}^{\prime}\right) \otimes E_{2}^{\prime}\left(t_{b}^{\prime}\right)\left|\psi^{\prime}(0)\right\rangle & =\left(I_{1} \otimes P^{\left|z^{\prime}+\right\rangle}\right)\left|\psi^{\prime}(0)\right\rangle, \\
& =\left|z^{\prime}+\right\rangle \otimes P^{\left|z^{\prime}+\right\rangle}\left|z^{\prime}-\right\rangle-\left|z^{\prime}-\right\rangle \otimes P^{\left|z^{\prime}+\right\rangle}\left|z^{\prime}+\right\rangle, \\
& =-\left|z^{\prime}-\right\rangle_{1} \otimes\left|z^{\prime}+\right\rangle_{2} .
\end{aligned}
$$

After the $A$-measurement, the state is:

$$
\begin{aligned}
E_{1}^{\prime}\left(t_{\mathrm{c}}^{\prime}\right) \otimes E_{2}^{\prime}\left(t_{\mathrm{c}}^{\prime}\right)\left|\psi^{\prime}(0)\right\rangle & =\left(P^{\left|x^{\prime}+\right\rangle_{1}} \otimes P^{\left|z^{\prime}+\right\rangle_{2}}\left|\psi^{\prime}(0)\right\rangle\right. \\
& =-\frac{1}{\sqrt{2}}\left|x^{\prime}+\right\rangle_{1} \otimes\left|z^{\prime}+\right\rangle_{2} .
\end{aligned}
$$

The differences in the state histories according to the two frames thus arise solely from a difference in the foliations used to define the evolving state of the extended system.

The scheme must be generalized to include multiple collapses occurring along the world-line of the same object, and also to include a combination of unitary and collapse evolution. Ideally one would like to see unitary evolution and collapse evolution as limiting cases of the same process, perhaps along the lines of Philip Pearle's continuous spontaneous localization model (CSL). (See Pearle, 1997 and references therein.) The scheme will also have to be generalized to a full fieldtheoretic context. Without committing ourselves as to the details of the collapse model, let us assume that there exist generalized evolution operators $E\left(t_{f} ; t_{i}\right)$ that take a system from a state on $t_{i}$ to its state on $t_{f}$, satisfying

$$
\begin{aligned}
& E(t ; t)=I, \\
& E\left(t_{f} ; t_{i}\right)=E\left(t_{f} ; t\right) E\left(t ; t_{i}\right) \text { for } t_{i} \leqslant t \leqslant t_{f} .
\end{aligned}
$$

For a system of $n$ individually localized subsystems $\left\{S_{k} \mid, k=1, \ldots, n\right\}$, relativity requires that the evolution operator factorize if the systems are spacelike separated during the interval $\left[t_{i}, t_{f}\right]$ :

$$
E\left(t_{f} ; t_{i}\right)=\bigotimes_{k=1}^{n} E_{k}\left(t_{f} ; t_{i}\right)
$$

Collapse evolution is not reversible; different initial states can collapse into the same final state. Therefore, $E\left(t_{1} ; t_{2}\right)$ will be undefined if $t_{1}<t_{2}$ and a collapse has taken place between $t_{1}$ and $t_{2}$. The transformation from one foliation to another,

$$
\left|\psi^{\prime}\left(t^{\prime}\right)\right\rangle=\Lambda_{1} E_{1}\left(t_{1}\left(t^{\prime}\right) ; t\right) \otimes \Lambda_{2} E_{2}\left(t_{2}\left(t^{\prime}\right) ; t\right)|\psi(t)\rangle,
$$

must therefore be handled with care. We cannot, for example, directly apply (51) to obtain the state on the hyperplane $t^{\prime}=t_{\mathrm{b}}^{\prime}$ from the state on $t=t_{\beta}$ or vice versa, as there is a collapse between the hyperplanes in each direction. This does not mean that the two states are unrelated, however, as they can both be obtained from 
the state on $t_{\alpha}$ as follows:

$$
\begin{aligned}
& \left|\psi\left(t_{\beta}\right)\right\rangle=E_{1}\left(t_{\beta} ; t_{\alpha}\right) \otimes E_{2}\left(t_{\beta} ; t_{\alpha}\right)\left|\psi\left(t_{\alpha}\right)\right\rangle, \\
& \left|\psi^{\prime}\left(t_{\mathrm{b}}^{\prime}\right)\right\rangle=\Lambda_{1} \otimes \Lambda_{2} E_{2}\left(t_{\gamma} ; t_{\alpha}\right)\left|\psi\left(t_{\alpha}\right)\right\rangle .
\end{aligned}
$$

In order to find a state defined along a hyperplane of constant $t^{\prime}$, one must in the general case apply the transformation to a state defined on a hyperplane of constant $t$ such that $t \leqslant t_{1}\left(t^{\prime}\right)$ and $t \leqslant t_{2}\left(t^{\prime}\right){ }^{7}$ With this proviso, it can readily be seen that a complete state history with respect to one foliation, together with a specification of the local evolution operators $\left\{E_{i}\right\}$, completely determines the state history with respect to any other foliation.

The relativity of entanglement entails that a system might be assigned a spin eigenstate by a state defined along one hyperplane and not be assigned any definite state at all by a state defined along another hyperplane passing through the very same instant on the system's worldline. One might wonder whether it is possible for a system to be assigned two different definite spin states at the same point on its worldline by two different hyperplanes intersecting the systems's worldline at that point. $^{8}$ The conditions (49)-(51) suffice to rule this out.

Let the spacelike hyperplanes $\beta$ and $c$ intersect at point $Q$ on $S_{1}$ 's worldline (refer to Fig. 1). Suppose that the states on $\beta$ and $c$ are, respectively,

$$
\begin{aligned}
& \left|\psi\left(t_{\beta}\right)\right\rangle=|u\rangle_{1} \otimes|v\rangle_{2}, \\
& \left|\psi^{\prime}\left(t_{\mathrm{c}}^{\prime}\right)\right\rangle=|\phi\rangle_{1} \otimes|\xi\rangle_{2} .
\end{aligned}
$$

Let $R$ be the intersection of the hyperplane $t=t_{\beta}$ with $S_{2}$ 's worldline, and let the hyperplane $t^{\prime}=t_{a}^{\prime}$ also intersect $S_{2}$ 's worldline at $R$. The segment of $S_{2}$ 's evolution that must be taken into account is the interval between $t^{\prime}=t_{\mathrm{a}}^{\prime}$ and $t^{\prime}=t_{\mathrm{c}}^{\prime}$ on $S_{2}$ 's worldline. All such points on $S_{2}$ 's worldline ate spacelike separated from $Q$, so, applying (50), we assume factorizable evolution and write:

$$
\left|\psi^{\prime}\left(t_{\mathrm{c}}^{\prime}\right)\right\rangle=\Lambda_{1} E_{1}\left(t_{1}\left(t_{\mathrm{c}}^{\prime}\right) ; t_{\beta}\right) \otimes \Lambda_{2} E_{2}\left(t_{2}\left(t_{\mathrm{c}}^{\prime}\right) ; t_{\beta}\right)\left|\psi\left(t_{\beta}\right)\right\rangle .
$$

But $t_{1}\left(t_{\mathrm{c}}^{\prime}\right)$ is just $t_{\beta}$, and so

$$
E_{1}\left(t_{1}\left(t_{\mathrm{c}}^{\prime}\right) ; t_{\beta}\right)=E_{1}\left(t_{\beta} ; t_{\beta}\right)=I_{1}
$$

Therefore

$$
\left|\psi^{\prime}\left(t_{\mathrm{c}}^{\prime}\right)\right\rangle=\left(\Lambda_{1} \otimes \Lambda_{2}\right) E_{2}\left(t_{2}\left(t_{\mathrm{c}}^{\prime}\right) ; t_{\beta}\right)\left|\psi\left(t_{\beta}\right)\right\rangle
$$

or

$$
|\phi\rangle_{1} \otimes|\xi\rangle_{2}=\Lambda_{1}|u\rangle_{1} \otimes \Lambda_{2} E_{2}\left(t_{2}\left(t_{\mathrm{c}}^{\prime}\right) ; t_{\beta}\right)|v\rangle_{2}
$$

\footnotetext{
${ }^{7}$ This restriction can be removed if the collapsed state contains 'tails' in which information about previous states in hidden. See Pearle (1997, p. 154).

${ }^{8} \mathrm{I}$ am grateful to Joseph Berkovitz for posing this as a problem.
} 
and so, up to a multiplicative constant, we must have

$$
|\phi\rangle_{1}=\Lambda_{1}|u\rangle_{1} \text {. }
$$

It follows that if $S_{1}$ is assigned a definite state vector by states defined along two hyperplanes passing through its worldline at a point $Q$, then the two state vectors will simply be the Lorentz transforms of each other, as consistency requires.

\section{Separability and locality}

So far, it has been argued that, with or without collapse, in a relativistic context the state of an extended system must be defined along a spacelike hypersurface of simultaneity, and that a state history must be defined with respect to a particular foliation of spacetime. Collapse evolution can be modelled by linear, local, nonunitary, stochastic evolution operators - at least, it can be so modelled within the scope of the approximate localizability assumption discussed in Section 1. The resulting state histories given with respect to alternate foliations are not conflicting accounts of different events but merely different descriptions of the same events, and a complete state history with respect to one foliation can be transformed into a state history with respect to another foliation via Eq. (51). A consequence of modelling collapse by linear, local, non-unitary evolution operators is the relativity of entanglement.

The probabilities governing the transitions of our local, stochastic collapse evolution operators are calculated in the usual quantum-mechanical way, that is, calculated with respect to the entire global state vector. The foliation dependence of the state vector thus entails foliation-dependence of these probabilities. The lesson of Bell's theorem is that these probabilities cannot be taken to factor into independent local probabilities, even if the quantum-mechanical state description were to be supplemented by some more complete description.

There is therefore a form of holism associated with the quantum-mechanical description of composite systems. This holism is exhibited by the fact that, in an entangled state, the composite system has properties not reducible to properties of the component subsystems. In the singlet state, for example, the sum of the spins of the two particles in any direction is zero. This is true in spite of the fact that the state does not assign definite values to the individual spins in any direction, and does not assign any spin state at all to the individual particles (since, for spin-1/2 systems, any spin state is a state of definite spin in some direction). Thus, the two individual spins are in a certain relation, that of summing to zero, that is not attributable to non-relational properties of the two individual systems, Teller (1989) has proposed the term relational holism for the view that there are relations that do not supervene on nonrelational properties of the things related. The relativity of entanglement can be regarded as one manifestation of this relational holism.

To regard a quantum-mechanical entangled state of systems that are spatially separated as a complete description entails a rejection of Einstein's principle of 
separability, which holds that the things dealt with in physics "claim an existence independent of one another, insofar as they 'lie in different parts of space'" (Einstein 1948, p. 321; translation by Don Howard, quoted in Howard 1985, p. 190). Howard $(1985,1989)$ has distinguished between this principle and another principle invoked by Einstein, the principle of locality, or absence of interaction between spatially separated systems. ${ }^{9}$ A natural translation of these principles into the language of quantum mechanics would be:

Separability: The state of a spatially extended system can always be written as a tensor product of local states.

Locality: The Hamiltonian of a spatially separated system can be written as a sum of local Hamiltonians.

The quantum-mechanical treatment of extended systems, on this reading, violates separability but is usually assumed to satisfy locality. ${ }^{10}$

A violation of Einstein locality in quantum theory would require a preferred notion of distant simultaneity for the state evolution, and, in fact, any such violation would permit superluminal signalling via suitable measurements carried out on suitably prepared states, as a choice of which observable was measured at $B$ would affect the statistics pertaining to measurements at $A$. That is, a violation of Einstein locality would entail a violation of the condition called "Locality" by Jarrett (1984, 1989) and "Parameter Independence" by Shimony (1986). An entangled state, which violates Einstein separability, violates, for suitable measurements, the condition called "Completeness" by Jarrett and "Outcome Independence" by Shimony.

The goal of the previous section was to make it plausible that, although a violation of Einstein separability is not what acquaintance with classical relativistic physics would lead one to expect, such a violation does not ipso facto constitute a violation of special relativity, if the entangled state can be written as sum of factorizable states, each undergoing purely local evolutions. Evolution governed by a Hamiltonian that is the sum of local Hamiltonians and represented by a unitary evolution operator that is a tensor product of local unitary evolution operators clearly counts as local evolution in this sense. Moreover, collapse evolution when the state is factorizable also clearly counts as local evolution. We have extended the picture to collapse evolution represented by operators that are tensor products of local collapse operators, operating on entangled states. This has some counterintuitive consequences, such as the relativity of entanglement, but clearly does not pick out a preferred foliation or reference frame.

\footnotetext{
${ }^{9}$ Einstein himself did not always clearly distinguish between the two principles. In the Dialectica article, for example, he refers to the two principles together as "Grundsatz II" (1948, p. 322). In the Schilpp volume, however, there is a passage that clearly distinguishes them: "(One can escape from this conclusion [that to the same real state of affairs one can assign two distinct $\psi$-functions] only by either assuming that the measurement of $S_{1}$ (telepathically) changes the real situation of $S_{2}$ or by denying independent real situations as such to things which are spatially separated from each other. Both alternatives appear to me entirely unacceptable.)" (Einstein, 1949, p. 85). It is likely that Einstein regarded locality and separability as two aspects of a single principle.

${ }^{10}$ Gordon Fleming has, however, argued for the introduction of non-local Hamiltonians. See Fleming (1988) and Fleming and Bennett (1989).
} 


\section{Maudlin's objections}

The sharpest critic of foliation-relative state evolution, and of foliation-relative collapse in particular, has been Maudlin (1994, 1996), and no defense of peaceful coexistence via foliation-relative state vector collapse can afford to ignore his objections. Maudlin's criticisms are chiefly directed at Fleming (1989), though he also cites Giovannini (1983), Aharonov and Albert (1984), and Dieks (1985). As has already been mentioned, the position defended in this paper differs in some aspects from Fleming's position. It seems, however, that Maudlin's criticisms are not affected by these differences, and hence that the present account, if it is to be regarded as a tenable position, must be capable of answering these objections. It is not the purpose of this section to discuss whether Maudlin has interpreted Fleming correctly, or whether his criticisms succeed as objections to Fleming's position; the issue at hand is whether his criticisms of Fleming succeed as objections to the position presented in this paper.

Maudlin asks (1994, p. 209),

Does it really make sense to say that a photon at a particular location has one polarization when thought of as lying along one hyperplane and another when thought of as lying along a different one? Or that a detection event is stochastic when one assigns it to one hyperplane but deterministic when assigned to another? The original difficulty in picking a hyperplane for wave collapse has been swamped by a radical generosity: in Fleming's theory there are wave collapses along an infinity of hyperplanes that pass through either detection event. Has Nature really been so profligate with collapse events?

The last question will be answered first: no, Nature has not been profligate with collapse events. There are not different collapse events corresponding to different foliations; the accounts given with respect to alternate choices of foliation are not descriptions of different events but different descriptions of the same events. In Example 1, there are two collapse events, whose descriptions in terms of state transitions depend upon the hypersurfaces of simultaneity one uses to define the instantaneous state of the evolving system.

This objection is raised in a sharper form in Maudlin's (1996). There he considers an example similar to our Example 1; in Maudlin's notation, the two measurement events are designated by $L$ and $R$, instead of $A$ and $B$. Maudlin considers two sets of hyperplanes: $\{\alpha, \beta, \gamma\}$ are hyperplanes of simultaneity in $\Sigma$, with $\alpha$ in the past of the $L$-measurement, $\beta$ lying in the future of the $L$-measurement and in the past of the $R$ measurement, and $\gamma$ lying in the future of both measurements; $\{a, b, c\}$ are hyperplanes of simultaneity in $\Sigma^{\prime}$, with $a$ in the past of the $R$-measurement, $b$ between the two measurements, and $c$ in the future of both (refer once more to Fig. 1). Thus, the measurement at $L$ lies between $\alpha$ and $\beta$, and between $b$ and $c$, while the measurement at $R$ lies between $\beta$ and $\gamma$, and between $a$ and $b$. In connection with 
this example, Maudlin (1996, p. 301) writes,

There seem to be four distinct collapses postulated so far: the collapse that transforms the $a$ singlet state into the $b R$-spin eigenstate (for both electrons); the collapse that transforms the latter wave function into the $L$-spin eigenstate for the $L$-electron and the $R$-spin eigenstate for the $R$ electron; the collapse that transforms the $\alpha$ singlet state into an $L$-spin eigenstate; and the collapse that transforms this latter into the $\gamma$ spin state. Using obvious notation, let us call these the $a-b, b-c, \alpha-\beta$, and $\beta-\gamma$ collapses.

Now, we certainly do have four state transitions here-in the state history according to $\Sigma$, we have a transition from the state defined on $\alpha$ to the state on $\beta$ and a transition from the state on $\beta$ to the state on $\gamma$, whereas in the state history according to $\Sigma^{\prime}$ we have a transition from the state on $a$ to the state on $b$ and a transition between the state on $b$ to the state on $c$. It would be misleading, however, to speak of these as four collapse events. Though one could, in a relativistic context, refer to a change in a (foliation-relative) instantaneous state of an extended system as an event, it is customary to save the word for local changes. That is, events, in special relativity, are associated with spacetime points, or else with spacetime regions that are sufficiently small that they can be regarded as points. One can - and I believe, should-regard collapse events as local events, in the sense discussed above, in Section 3. It is the same collapse event, namely, the collapse at $L$ that occurs when the measurement at $L$ takes place, that induces the $\alpha-\beta$ transition and the $b-c$ transition. Similarly, it is the same collapse event, the one at $R$, that induces the $\beta-\gamma$ transition and the $a-b$ transition.

Maudlin's objection can be cast in the form of a dilemma. Either the state histories along each foliation agree as to the macroscopic outcomes of experiments, or they don't. If all foliations agree on the outcome of experiments, this is a coincidence inexplicable on the basis of the dynamics of the theory, which only connect states within a foliation (Maudlin, 1996, p. 301). If, on the other hand, the state histories do not agree on such outcomes, "[t]he wave functions on each family of hyperplanes would then completely decouple, yielding an independent world for each foliation" (p. 302).

The first horn of the dilemma, according to Maudlin, leaves us with unexplained correlations. While quantum mechanics accounts for correlations in outcomes between the $\alpha-\beta$ collapse and the $\beta-\gamma$ collapse, and for correlations between the $a-b$ collapse and the $b-c$ collapse, "there is still an unexplained correlation. The $a-b$ collapse is correlated not only with the $b-c$ collapse, but also with the $\alpha-\beta$ collapse" (1996, p. 301). The answer to this objection should be obvious: the $b-c$ collapse-that is, the collapse that induces the transition from the $b$-state to the $c$-state - is the same event as the $\alpha-\beta$ collapse. Thus a correlation between the $a-b$ collapse and the $b-c$ collapse is ipso facto a correlation between the $a-b$ collapse and the $\alpha-\beta$ collapse. 
The objection presupposes that states defined along different foliations are ontologically independent of each other.

The wave function on a hyperplane is, as it were, ontologically atomic. Wave functions defined on hyperplanes in the same family can be related by a dynamics which uses the family as a substitute for absolute time, but relations among wave functions from different families are obscure at best (1996, p. 302).

This supposed ontological independence does not exist, however. A factorizable state defined on a hypersurface $\sigma$ is merely one way of splicing together local parts into an instantaneous global state; entangled states are superpositions of such splicings. A complete state history given with respect to one foliation uniquely determines the state history with respect to any other foliation; the relations between states defined with respect to different foliations are given by Eq. (51).

Let us return to the first question asked by Maudlin in the paragraph quoted at the beginning of this section: "Does it really make sense to say that a photon at a particular location has one polarization when thought of as lying along one hyperplane and another when thought of as lying along a different one?" Maudlin is here referring to the relativity of entanglement. The way that Maudlin phrases the question is misleading, though; Maudlin tends to call being entangled with another photon a "polarization state" of an individual photon. When a pair of systems is in an entangled state, however, quantum mechanics assigns no state vectors to the individual systems; the individual photons, when the system is in an entangled state, do not have quantum-mechanical polarization states.

According to Maudlin, the relativity of entanglement not only shocks commonsense intuitions, but also "shocks intuitions which are formed by acquaintance both with relativity and with non-relativistic quantum mechanics” (1994, p. 209).

The language of relations, of the photon having a property relative to a hypersurface, makes the situation sound a bit more prosaic. Socrates can be tall relative to Theatetus and short relative to Plato without thereby engendering any paradoxes or puzzlement. But hyperplane dependence is more unfamiliar, and stranger, than this analogy suggests. First, it is obvious that tallness and shortness are relations, not properties that an object could have in and of itself alone. If the universe contained but one object, that object could not in principle be either short or tall. But polarization is not such an intrinsically relational matter. In a universe with but one photon, the photon could have a definite state of polarization. Indeed, one has the strong intuition that whether or not a photon is polarized should be a matter of the intrinsic state of it, independent of any considerations about hyperplanes. (p. 210)

In this context, to say that a photon is "polarized" means that it has its own polarization state, and to say that it is not polarized means, not that it has a state 
vector that is a superposition of distinct polarization states, but that it is entangled with some other system and so is not ascribed its own state vector at all. The last sentence of the quoted paragraph, then, says that one has the strong intuition that whether or not a photon is entangled with some other system should be a matter purely of the intrinsic state of it, and not a matter of its relations to other objects. However, it is not clear that intuition runs in this direction at all. ${ }^{11}$

According to Maudlin (1994, p. 212),

The theory of hyperplane dependence contradicts Einstein's supposition [of separability] in a particularly radical fashion. It is not just that there are some relations which don't supervene on the intrinsic state of the relata but that the relata don't have any intrinsic state at all. It is not just that the whole is more than the sum of the parts but that the parts can't even be defined apart from the whole.

This is exactly right, except that it applies equally well to quantum mechanics in Galilean spacetime, in which there is a distinguished foliation of spacetime and hence a unique state history, as long as one accepts that the state vector of a system is a complete description of the system. It is not the hyperplane dependence of state vectors that violates Einstein separability, but the supposition that the state vector is all there is, and hence that the components of a system in an entangled state do not have individual states of their own. It is not collapse, foliation-relative or otherwise, that Maudlin is objecting to in this passage, but entanglement construed ontologically.

There is, however, something new when one adds collapse to the picture of foliation-relative evolution of entangled states, namely, the relativity of entanglement. One does, I believe, have a strong intuition that, if $S_{1}$ is not entangled with $S_{2}$, then this circumstance is a matter of the intrinsic state of $S_{1}$, and it is this intuition

\footnotetext{
${ }^{11}$ This discussion presumes that the state of a system is represented by a state vector. If density operators are used instead, then one can always associate with the component systems the reduced density operators $\rho_{1}$ and $\rho_{2}$. Then the state of the composite system is factorizable if and only if $\rho_{12}=\rho_{1} \otimes \rho_{2}$. If the evolution from one hyperplane to another is unitary, then the reduced density operators along different hyperplanes all passing through the same point on a localized system's worldline are just the Lorentz transforms of each other: $\rho_{i}^{\prime}\left(t_{i}^{\prime}(t)\right)=\Lambda_{i} \rho_{i}(t) \Lambda_{i}^{\dagger}$. This is not the case if collapse evolution is included. In the absence of collapse, one could, perhaps, think of the reduced density operators as describing intrinsic states of the subsystem. These reduced operators will be pure states if the global state is factorizable and improper mixtures if the global state is entangled. On such an account, there is some plausibility to the claim that whether or not a photon is polarized is a matter of the intrinsic state of it. In a footnote Maudlin $(1994,222$, fn. 10) remarks, "In referring to the "polarization state" of the photon I do not mean to presuppose that the photon by itself necessarily has any state at all. There is some dispute about whether a member of a pair in an entangled state should itself be ascribed a so-called reduced state or should only be ascribed a joint state with the second photon. I incline to the latter view, but the issue is not important here. Whatever one wants to say about the photon, let that be its 'state'." It seems, however, that any plausibility there is in the view that whether or not a photon is entangled with another is a matter of the intrinsic state of it stems from regarding reduced density operators as representations of the intrinsic states of subsystems, even when the global state is entangled.
} 
that seems to be the one that Maudlin is alluding to. Acceptance of collapse occurring as part of a foliation-relative state evolution entails regarding this intuition, formed, as Maudlin points out, by acquaintance with relativity and nonrelativistic quantum mechanics, as mistaken. To invoke an anthropocentric analogy: if marriage is a relational state, so too is divorce!

The second question in the paragraph from Maudlin quoted at the beginning of this section asks: "Does it really make sense to say... that a detection event is stochastic when one assigns it to one hyperplane but deterministic when assigned to another?" As noted above, reference frames may disagree on which collapse events are chance events and which have predetermined outcomes. This is a consequence of the fact that, though collapse events can be regarded as local events, the probabilities assigned to the various possible outcomes of this local event are calculated with respect to the globally defined state vector, and hence are foliation-relative. To be predetermined is to have probability one for some outcome, and zero for all others, and an event may be assigned probability one by a state defined along one hyperplane passing through the event, and a probability of less than one by a state defined along another hyperplane passing through the event. This is odd. One would expect, in any stochastic theory, that all facts that are relevant to the probabilities of outcomes of a given event be confined to the past light-cone of the event. It is the message of Bell's theorem, however, that the probabilities assigned to outcomes of measurements at spacelike separation by quantum-mechanical entangled states cannot be reduced to products of independent local probabilities. It is in this sense that quantum theory is non-local. ${ }^{12}$

Although this runs contrary to intuitions trained by exposure to relativity theory, it is not clear that this feature of quantum-mechanical probabilities constitutes an actual conflict with relativity, so long as every reference frame agrees on the probability assigned to any set of outcomes of a set of spacelike-separated measurement events. This ensures that the statistics of a repeated series of measurements will not distinguish between reference frames (or between alternate foliations).

\section{Transformation of wave functions}

Let us return to Example 2. We want to write the (non-local) state of the particle as a tensor product of local states. We can do this via an occupation-number representation for the states of the three boxes. Let $\left|n_{1} n_{2} n_{3}\right\rangle=\left|n_{1}\right\rangle_{1} \otimes\left|n_{2}\right\rangle_{2} \otimes\left|n_{3}\right\rangle_{3}$ be a state in which there are $n_{1}$ particles in box $1, n_{2}$ particles in box 2 ,

\footnotetext{
${ }^{12}$ However, quantum mechanics is not non-local in the sense of a violation of Einstein's principle of locality. The non-local nature of quantum-mechanical probabilities is due, rather, to the entanglement of spatially separated systems, which is a violation of Einstein's principle of separability.
} 
and $n_{3}$ particles in box $3 .^{13}$ Then we have

$$
\begin{aligned}
& \left|\mathbf{x}_{1}\right\rangle=|100\rangle=|1\rangle_{1} \otimes|0\rangle_{2} \otimes|0\rangle_{3}, \\
& \left|\mathbf{x}_{2}\right\rangle=|010\rangle=|0\rangle_{1} \otimes|0\rangle_{2} \otimes|0\rangle_{3}, \\
& \left|\mathbf{x}_{3}\right\rangle=|001\rangle=|0\rangle_{1} \otimes|0\rangle_{2} \otimes|1\rangle_{3} .
\end{aligned}
$$

The initial state $|\alpha\rangle$ is given by

$$
|\alpha\rangle=|100\rangle+|010\rangle+|001\rangle \text {. }
$$

The state $|\alpha\rangle$ is, therefore, an entangled state of the local box-states.

The sequence of box-openings is given by

$$
E(t)|\alpha\rangle=E_{1}(t) \otimes E_{2}(t) \otimes E_{3}(t)|\alpha\rangle .
$$

where

$$
\begin{aligned}
& E_{1}(t)= \begin{cases}I_{1} & \text { before box } 1 \text { is opened. } \\
P^{|0\rangle_{1}} & \text { after box } 1 \text { is opened. }\end{cases} \\
& E_{2}(t)= \begin{cases}I_{2} & \text { before box } 2 \text { is opened. } \\
P^{|0\rangle_{2}} & \text { after box } 2 \text { is opened. }\end{cases} \\
& E_{3}(t)=I_{3} .
\end{aligned}
$$

$E_{1}^{\prime}\left(t^{\prime}\right), E_{2}^{\prime}\left(t^{\prime}\right), E_{3}^{\prime}\left(t^{\prime}\right)$ are defined similarly. With respect to $\Sigma^{\prime}$, the state history is given by

$$
E^{\prime}\left(t^{\prime}\right)\left|\alpha^{\prime}\right\rangle=E_{1}^{\prime}\left(t^{\prime}\right) \otimes E_{2}^{\prime}\left(t^{\prime}\right) \otimes E_{3}^{\prime}\left(t^{\prime}\right)\left|\alpha^{\prime}\right\rangle
$$

The two state histories are, therefore, Lorentz transforms of one another, in much the same way as the state histories discussed in Example 1.

A few comments are in order about this treatment of the example. First, the singleparticle state $|\alpha\rangle$ is an entangled state of the local box-states. Second, the state $\left|\mathbf{x}_{1}\right\rangle$ is not a local state of box 1 alone, but is a global state of the three boxes; it is a state in which there is one particle in box 1 and none in either of the other two boxes. As the boxes are located some distance apart, such states are defined only relative to a hypersurface of simultaneity. A general state in which, on hypersurface $\sigma$, there is exactly one particle somewhere in the three boxes is given by

$$
|\psi\rangle_{\sigma}=\psi_{\sigma}\left(\mathbf{x}_{1}\right)\left|\mathbf{x}_{1}\right\rangle_{\sigma}+\psi_{\sigma}\left(\mathbf{x}_{2}\right)\left|\mathbf{x}_{2}\right\rangle_{\sigma}+\psi_{\sigma}\left(\mathbf{x}_{3}\right)\left|\mathbf{x}_{3}\right\rangle_{\sigma} .
$$

Since the $\left|\mathbf{x}_{i}\right\rangle_{\sigma}$ 's are not local states but rather global states on $\sigma$, the values of $\psi_{\sigma}\left(\mathbf{x}_{i}\right)$ are not local quantities but are, rather, defined in terms of the entire state on $\sigma$. There is no reason for $\psi_{\sigma}\left(\mathbf{x}_{i}\right)$ and $\psi_{\sigma^{\prime}}\left(\mathbf{x}_{i}^{\prime}\right)$ to coincide for distinct hypersurfaces of simultaneity $\sigma$ and $\sigma^{\prime}$, even if the two hypersurfaces intersect at $\mathbf{x}_{i}$.

\footnotetext{
${ }^{13}$ In light of the restrictions placed by quantum field theory on localization, this representation can only be regarded as an approximation to the real state.
} 
Suppose that, in Example 2, box 2 had been opened first, and the particle had been detected there. This transition would be modelled by the collapse evolution operator

$$
E(t)=I_{1} \otimes E_{2}(t) \otimes I_{3},
$$

where

$$
E_{2}(t)= \begin{cases}I_{2} & \text { before box } 2 \text { is opened } \\ P^{|1\rangle_{2}} & \text { after box } 2 \text { is opened. }\end{cases}
$$

Suppose the initial state at $t=0$ (that is, on the hyperplane $t=0$ ) is given by

$$
|\psi(0)\rangle=\psi_{(t=0)}\left(\mathbf{x}_{1}\right)|100\rangle+\psi_{(t=0)}\left(\mathbf{x}_{2}\right)|010\rangle+\psi_{(t=0)}\left(\mathbf{x}_{3}\right)|001\rangle .
$$

After the box opening, this becomes

$$
\begin{aligned}
\left(I_{1}\right. & \left.\otimes P^{|1\rangle_{2}} \otimes I_{3}\right)|\psi(0)\rangle \\
& =\left(I_{1} \otimes P^{|1\rangle_{2}} \otimes I_{3}\right)\left(\psi_{(t=0)}\left(\mathbf{x}_{1}\right)|100\rangle+\psi_{(t=0)}\left(\mathbf{x}_{2}\right)|010\rangle+\psi_{(t=0)}\left(\mathbf{x}_{3}\right)|001\rangle\right), \\
& =\psi_{(t=0)}\left(\mathbf{x}_{2}\right)|010\rangle .
\end{aligned}
$$

And so, on a hyperplane after the opening, say, $t=1$, we have

$$
\begin{aligned}
& \psi_{(t=1)}\left(\mathbf{x}_{1}\right)=0, \\
& \psi_{(t=1)}\left(\mathbf{x}_{2}\right)=\psi_{(t=0)}\left(\mathbf{x}_{2}\right), \\
& \psi_{(t=1)}\left(\mathbf{x}_{3}\right)=0 .
\end{aligned}
$$

We have the seemingly paradoxical fact that the local act of opening box 2 instantaneously reduces $\psi\left(\mathbf{x}_{1}\right)$ and $\psi\left(\mathbf{x}_{3}\right)$ to zero. Yet the air of paradox disappears when we recall that these are not locally defined quantities but coefficients of expansion of the state along a specified hyperplane in terms of globally defined states on that hyperplane. Insofar as there is a wave function at all, whose square gives a probability density for the location of a single particle (and this must, in a relativistic context, be regarded merely as an approximation), it is a foliation-relative object: not a function mapping spacetime points onto numbers but a functional taking both a spacelike hypersurface and a point on that hypersurface as arguments (Tomonaga, 1946; Schwinger, 1948). There is no contradiction, therefore, in the claim that the collapse of the wave function is simultaneous with respect to every reference frame and, in general, with respect to any foliation of spacetime into hypersurfaces of simultaneity.

\section{A final comment on relativistic collapse models}

Beginning with the work of Ghirardi, Rimini, and Weber (1986), a number of models for collapse dynamics have been proposed. One of the most attractive of these is Philip Pearle's (1997) continuous spontaneous localization model (CSL). Pearle $(1999 \mathrm{a}, \mathrm{b})$ has also done work on extending CSL to relativistic quantum theories. There are difficulties associated with such an extension, but these have to do 
with vacuum divergences; there is a tendency for the model to produce an infinite number of particles per unit time from the vacuum. This issue seems closely related to the divergences that crop up in quantum field theory without collapse, and is not obviously related to the arguments considered in this paper. Thus, while we do not currently have a clearly unproblematic relativistic collapse model, neither do we have strong reason to believe that no such model can be constructed.

\section{Acknowledgements}

The author would like to thank Frank Arntzenius, Joseph Berkovitz, Rob Clifton, Michael Dickson, Michael Redhead, Abner Shimony, and an anonymous referee for helpful discussions and comments.

\section{References}

Aharonov, Y., \& Albert, D. (1980). States and observables in relativistic quantum field theories. Physical Review D, 21, 3316-3324.

Aharonov, Y., \& Albert, D. (1981). Can we make sense out of the measurement process in relativistic quantum mechanics? Physical Review D, 24, 359-370.

Aharonov, Y., \& Albert, D. (1984). Is the usual notion of time evolution adequate for quantummechanical systems?: II. Relativistic considerations. Physical Review D, 29, 228-234.

Clifton, R., Pagonis, C., \& Pitowsky, I. (1992). Relativity, quantum mechanics, and EPR. In D. Hull, M. Forbes, \& K. Okruhlik (Eds.), PSA 1992, Vol. 1 (pp. 114-128). East Lansing, MI: Philosophy of Science Association.

Cushing, J. T. (1994). Quantum mechanics: Historical contingency and the Copenhagen hegemony. Chicago: The University of Chicago Press.

Dickson, M., \& Clifton, R. (1998). Lorentz-invariance in modal interpretations. In D. Dieks \& P. E. Vermaas (Eds.), The modal interpretation of quantum mechanics (pp. 9-47). Dordrecht: Kluwer Academic Publishers.

Dieks, D. (1985). On the covariant description of wavefunction collapse. Physics Letters, 108 A, 379-383.

Dirac, P. A. M. (1933). The Lagrangian in quantum mechanics. Physikalische Zeitschrift der Sowjetunion, 3, 64-72. Reprinted in Schwinger (Ed.) (1958) Selected papers on quantum electrodynamics. New York: Dover Publications. (pp. 312-320).

Eberhard, P. H. (1978). Bell's theorem and the different concepts of locality. Il Nuovo Cimento, 46B, 392-419.

Einstein, A. (1948). Quanten-Mechanik and Wirklichkeit. Dialectica, 2, 320-323.

Einstein, A. (1949). Autobiographical notes. In P. A. Schilpp (Ed.), Albert Einstein: Philosopher-scientist (pp. 3-94). La Salle, IL: Open Court.

Fleming, G. N. (1986). On a Lorentz invariant quantum theory of measurement. In D. M. Greenberger (Eds.), New techniques and ideas in quantum measurement theory. Annals of the New York Academy of Sciences, 480, 574-575.

Fleming, G. N. (1988). Hyperplane-dependent quantized fields and Lorentz invariance. In H. R. Brown \& R. Harré (Ed.), Philosophical foundations of quantum field theory (pp. 93-115). Oxford: Clarendon Press.

Fleming, G. N. (1989). Lorentz invariant state reduction, and localization. In A. Fine \& M. Forbes (Eds.), PSA 1988, Vol. 2 (pp. 112-126). East Lansing, MI: Philosophy of Science Association.

Fleming, G. N. (1996). Just how radical is hyperplane dependence? In Clifton (Ed.), Perspectives on quantum reality: non-relativistic, relativistic, and field-theoretic. Dordrecht: Kluwer Academic Publishers. (pp. 11-28). 
Fleming, G. N., \& Bennett, H. (1989). Hyperplane dependence in relativistic quantum mechanics. Foundations of Physics, 19, 231-267.

Fleming, G. N., \& Butterfield, J. (2000). Strange positions. In J. Butterfield \& C. Pagonis (Ed.), From physics to philosophy (pp. 108-165). Cambridge: Cambridge University Press.

Ghirardi, G. C., \& Pearle, P. (1990). Elements of physical reality, nonlocality and stochasticity in relativistic dynamical reduction models. In A. Fine, M. Forbes, \& L. Wessels (Eds.), PSA 1990, Vol. 2 (pp. 35-47). East Lansing, MI: Philosophy of Science Association.

Ghirardi, G. C., Rimini, A., \& Weber, T. (1980). A general argument against superluminal transmission through the quantum mechanical measurement process. Lettere al Nuovo Cimento, 27, 294-298.

Ghirardi, G. C., Rimini, A., \& Weber, T. (1986). Unified dynamics of microscopic and macroscopic systems. Physical Review D, 34, 470-491.

Giovannini, N. (1983). Relativistic kinematics and dynamics: A new group theoretical approach. Helvetica Physica Acta, 56, 1002-1023.

Howard, D. (1985). Einstein on locality and separability. Studies in History and Philosophy of Science, 16, $171-201$.

Howard, D. (1989). Holism, separability, and the metaphysical implications of the Bell experiments. In Cushing \& McMullin (Eds.), Philosophical consequences of quantum theory: Reflections on Bell's theorem. Notre Dame, IN: University of Notre Dame Press. (pp. 224-253).

Jarrett, J. (1984). On the physical significance of the locality conditions in the Bell arguments. Nôus, 18, $569-589$.

Jarrett, J. (1989). Bell's theorem: A guide to the implications. In Cushing \& McMullin (Eds.), Philosophical consequences of quantum theory: Reflections on Bell's theorem. Notre Dame, IN: University of Notre Dame Press. (pp. 60-79).

Malament, D. B. (1996). In defense of dogma: Why there cannot be a relativistic quantum mechanics of (localizable) particles. In R. Clifton (Ed.), Perspectives on quantum reality: non-relativistic, relativistic, and field-theoretic. Dordrecht: Kluwer Academic Publishers. (pp. 1-10).

Maudlin, T. (1994). Quantum non-locality and relativity: Metaphysical intimations of modern physics. Cambridge, MA: Blackwell Publishers.

Maudlin, T. (1996). Space-time in the quantum world. In J. T. Cushing, A. Fine, \& S. Goldstein (Eds.), Bohmian mechanics and quantum theory: An appraisal (pp. 285-307). Dordrecht: Kluwer Academic Publishers.

Pearle, P. (1989). Combining stochastic dynamical state-vector reduction with spontaneous localization. Physical Review A, 39, 2277-2289.

Pearle, P. (1997). Tales and tails and stuff and nonsense. In Cohen, et al. (Eds.), Potentiality, entanglement, and passion-at-a-distance: Quantum mechanical studies for Abner Shimony, Vol. 1, Dordrecht: Kluwer Academic Publishers. (pp. 143-156).

Pearle, P. (1999a). Collapse models. In Breuer \& Petruccione (Eds.), Open systems and measurement in relativistic quantum theory: Proceedings of the workshop held at the Istituto Italiano per gli Studi Filosofici. Naples, April 3-4, 1998. Berlin: Springer. (pp. 195-234). (Also available at xxx.lanl.gov e-print archive, quant-ph/9901077.)

Pearle, P. (1999b). Relativistic collapse model with tachyonic features. Physical Review A, 59, 80-101. Also available at xxx.lanl.gov e-print archive, quant-ph/9902046.

Percival, I. (1998). Quantum state diffusion. Cambridge: Cambridge University Press.

Schutz, B. F. (1990). A first course in general relativity. Cambridge: Cambridge University Press.

Schwinger, J. (1948). Quantum electrodynamics I. A covariant formulation. Physical Review, 74, 1439-1461.

Shimony, A. (1978). Metaphysical problems in the foundations of quantum mechanics. International Philosophical Quarterly, 18, 3-17.

Shimony, A. (1986). Events and processes in the quantum world. In R. Penrose \& C. Isham (Eds.), Quantum concepts in space and time (pp. 182-203). Oxford: Oxford University Press. Reprinted in Shimony (1993) (pp. 140-162).

Shimony, A. (1993). Search for a naturalistic world view, Vol. II, Natural Science and metaphysics, Cambridge: Cambridge University Press. 
Stein, H. (1991). On relativity theory and the openness of the future. Philosophy of Science, 58, 147-167.

Teller, P. (1989). Relativity, relational holism, and the Bell inequalities. In Cushing \& McMullin (Eds.), Philosophical consequences of quantum theory: Reflections on Bell's theorem. Notre Dame, IN: University of Notre Dame Press. (pp. 208-223).

Tomonaga, S. (1946). On a relativistically invariant formulation of the quantum theory of wave fields. Progress of Theoretical Physics 1, 1-13. Reprinted in Schwinger, Ed. (1958) Selected papers on quantum electrodynamics. New York: Dover Publications (pp. 156-168).

von Neumann, J. (1932/1955). Mathematical foundations of quantum mechanics. Tr. R. T. Bayer (Ed.). Princeton: Princeton University Press.

Weinberg, S. (1995). The quantum theory of fields. Vol. I. Foundations, Cambridge: Cambridge University Press. 